\title{
A new variable sampling size and interval synthetic and runs-rules schemes to monitor the process mean of autocorrelated observations with measurement errors
}

\author{
Sandile Charles Shongwe $^{a^{*}}$ and Jean-Claude Malela-Majika ${ }^{a}$
}

${ }^{a}$ Department of Statistics, College of Science, Engineering and Technology, University of South Africa; Pretoria, South Africa

\begin{tabular}{l}
\hline C H R O N I C L E \\
\hline Article history: \\
Received October 212019 \\
Received in Revised Format \\
April 82020 \\
Accepted April 172020 \\
Available online \\
April 172020 \\
\hline Keywords: \\
Autocorrelation \\
Measurement errors \\
Multiple measurements \\
Runs-rules \\
Skipping sampling strategy \\
Synthetic chart \\
Variable sampling size and \\
interval (VSSI)
\end{tabular}

\section{Introduction}

Since the vast majority of the contributions in the statistical process monitoring (SPM) field assumes that observations are: (i) i.i.d. (independent and identically distributed), and (ii) correspond to perfect measurements, the experience in SPM application shows that this is usually not the case in real life applications. That is, (i) within-sample autocorrelation (see the review by Prajapati and Singh (2012)) and (ii) measurement errors (see the review by Maleki et al. (2017)) are well-known to negatively affect the statistical efficiency of any monitoring scheme. Consequently, more efficient monitoring schemes are required to be able to quickly distinguish between a process that is in a state of in-control (IC) and the one in a state of out-of-control (OOC) when the process is under a combined effect of autocorrelation and measurement errors. A process is said to be IC (OOC) when it is under the effect of common (assignable) causes of variability, respectively. Supplementary runs-rules and synthetic schemes have been documented in the SPM literature to be one of the most efficient Shewhart-type monitoring schemes

\footnotetext{
* Corresponding author

E-mail: sandile@tuks.co.za (S. C. Shongwe)

2020 Growing Science Ltd. doi: $10.5267 /$ j.ijiec. 2020.4 .003
} 
for i.i.d. observations; see the reviews by Koutras et al. (2007) and Rakitzis et al. (2019), respectively. Hence, in this paper, efficient runs-rules and synthetic monitoring schemes are proposed to monitor the process mean under the combined effect of autocorrelation and measurement errors. Before introducing these new monitoring schemes, a few preliminaries are discussed below.

Firstly, numerous publications have shown that monitoring schemes that incorporate a variable sample size and sampling interval (VSSI) approach yield better OOC performance than their corresponding fixed sample size and sampling interval (FSSI) counterparts; see the review by Psarakis (2015). A majority of research works in the SPM literature based on the VSSI approach have been implemented for i.i.d. observations, see for instance Prabhu et al. (1994), Costa (1997), Celano et al. (2006), Jensen et al. (2008), Noorossana et al. (2015, 2016). Note though, Sabahno and Amiri (2017) studied the effect of measurement errors on the VSSI $\bar{X}$ scheme. However, so far, no research work exists in the SPM literature that monitors the combined effect of autocorrelation and measurement errors using a VSSI approach. Secondly, combining the operations of the basic Shewhart $\bar{X}$ scheme with the conforming runlength (CRL) scheme yields a synthetic $\bar{X}$ scheme; which was first proposed by Wu and Spedding (2000). The $C R L$ is defined as the number of sampling points observed between two consecutive nonconforming samples, inclusive of the nonconforming sample at the end. Note that a sample plots on a conforming region when it is under the influence of common causes of variation only; however, when it plots on a nonconforming region, it implies that it has some assignable causes of variation present. Hence, a synthetic monitoring scheme gives an OOC signal when the $C R L$ value is significantly small, say, $C R L$ $\leq H$, where $H$ is a positive integer greater than 0 . That is, the main difference between a basic Shewhart scheme and a synthetic scheme is that the basic Shewhart scheme issues an OOC signal at the first sample point that falls on the nonconforming region; however, synthetic scheme waits until a second sample point falls on the nonconforming region and if these two nonconforming samples are relatively close to each other, so that $C R L \leq H$, then the CRL scheme signals and thus, an OOC signal is triggered. For some recent works on synthetic monitoring schemes for i.i.d. observations, see Haq (2019), Khaw et al. (2019), Tran et al. (2019), Malela-Majika (2019), Raza et al. (2019), Haq and Khoo (2019), etc. However, for autocorrelated observations, see Hu and Sun (2015) and Shongwe et al. (2019a); and finally for autocorrelated observations with measurement errors using FSSI approach, see Shongwe et al. (2020a). It is important for synthetic schemes to evaluate both zero- and steady-state run-length properties, see Davis and Woodall (2002). The zero- and steady-state modes are used to characterize short- and longterm run-length properties of a monitoring scheme, respectively. That is, a zero-state run-length is a number of sampling points at which the scheme first signals given it begins in some specific initial state, whereas a steady-state run-length is a number of sampling points at which the scheme first signals given that the process begins and stays IC for a very long time, then at some random time, an OOC signal is observed.

Thirdly, supplementary runs-rules have been in use since the 1950s to improve the performance of Shewhart schemes; however, they have also been used to improve memory-type monitoring schemes more recently. For more recent research works on runs-rules monitoring schemes for i.i.d. observations, see Adeoti and Malela-Majika (2020), Shongwe et al. (2019b), Maravelakis et al. (2019), Mehmood et al. (2019), Chew et al. (2019), etc. However, for autocorrelated observations, see Singh and Prajapati (2014), Lee and Khoo (2018), and Shongwe and Malela-Majika (2019); and finally for autocorrelated observations with measurement errors, see Shongwe et al. (2020b). Fourthly, the synthetic-type schemes were shown to have four categories in Rakitzis et al. (2019). Shongwe and Graham (2016) showed that the improved 2-of- $(H+1)$ runs-rules schemes also have four categories. These are termed: non-sidesensitive (NSS), standard side-sensitive (SSS), revised side-sensitive (RSS) and modified side-sensitive (MSS) designs. The MSS design was shown to outperform the other three types of designs for the synthetic and runs-rules schemes. Hence, to gain maximum OOC performance improvement, in this paper, the focus is on the MSS design only. Finally, the MSS synthetic and runs-rules schemes with the VSSI approach for monitoring the process mean are denoted by VSSI-Synth and VSSI-Runs schemes, 
respectively. Therefore, the main objective of this paper is to improve the FSSI Shewhart $\bar{X}$ scheme for monitoring the process mean under a combined effect of autocorrelation and measurement errors (discussed in Costa and Castagliola, 2011) by using the new VSSI-Synth and VSSI-Runs monitoring schemes. That is, a dedicated Markov chain matrix is constructed which incorporates the following in the probability values: (i) VSSI approach, (ii) the MSS design for the charting regions, (iii) the first-order autoregressive (i.e. AR(1)) model with a constant standard deviation (to account for autocorrelation), (iv) the linearly covariate error model with a constant standard deviation (to account for the measurement errors). To reduce the negative effect of the latter two factors, the skipping sampling strategy and multiple measurements strategy are implemented; see the discussion of these strategies in Aslam and Ali (2019), and Linna and Woodall (2001), respectively.

The rest of the paper is structured as follows: The theoretical properties of the basic $\bar{X}$ scheme with a VSSI design for a process with a combined effect of autocorrelation and measurement errors is discussed in Section 2. In Section 3, the operation and run-length properties of the new VSSI-Synth and VSSIRuns monitoring schemes are discussed; with the construction of a dedicated Markov chain matrix done in the Appendix. The corresponding empirical discussion is done in Section 4. An implementation example is given in Section 5. Concluding remarks are given in Section 6.

\section{Autocorrelated data with measurement errors for the $\bar{X}$ scheme with VSSI design}

Improving from Alwan and Radson (1992) discussion on monitoring an AR(1) process using an $\bar{X}$ scheme, Costa and Castagliola (2011) integrated the latter process with the skipping $s$ observations before sampling to form a rational subgroup (denoted as $s$-skip strategy, where $s$ is a positive integer) as a remedial approach in reducing the effect of autocorrelation and showed that the process remains an $\operatorname{AR}(1)$; however, with parameter $\phi^{s+1}$, where $\phi$ is a specified parameter called a level of autocorrelation, with $|\phi|<1$. In an effort to further improve the performance of the $\bar{X}$ scheme, the use of the VSSI design instead of the FSSI design by Costa and Castagliola (2011) is introduced in the model. Consequently, the sequence of observations from an $\mathrm{AR}(1)$ process are then defined as $\left\{Y_{t,(s+1) i-s}: t \geq 0 ; i=1,2, \ldots, n_{r}\right\}$ with parameter $\phi^{s+1}$ are given by

$$
Y_{t,(s+1) i-s}-\mu_{0}=\phi^{s+1}\left(Y_{t,((s+1) i-s)-s-1}-\mu_{0}\right)+\varepsilon_{i}^{\prime}
$$

where $t$ denotes the sample number or a sampling point taken at every $d_{r}$ time intervals, where $r=1$ (2) implies that a long (short) sampling interval at that specific $t$, respectively, with $d_{1}>d_{2}$. Also, assume that $n_{1}\left(n_{2}\right)$ denotes the small (large) sample size at that specific sample number $t$, respectively, with $n_{1}<n_{2}$. Moreover, $\varepsilon_{i}^{\prime}=\varepsilon_{i}+\phi \varepsilon_{i-1}+\phi^{2} \varepsilon_{i-2}+\cdots+\phi^{s} \varepsilon_{i-s}, \varepsilon_{i}$ are i.i.d. normal $\left(0, \sigma_{\varepsilon}\right)$ random variables. The nominal IC mean and standard deviation process parameters are denoted by $\mu_{0}$ and $\sigma_{0}$, respectively, where $\sigma_{0}=\frac{\sigma_{\varepsilon}}{\sqrt{1-\phi^{2}}}$, and without loss of generality, assume $\sigma_{\varepsilon}=1$. Next, assume that the $Y_{t,(s+1) i-s}$ are not directly observable, but can only be assessed from the results $\left\{X_{t,(s+1) i-s, j}: t \geq 0 ; i=\right.$ $\left.1,2, \ldots, n_{r} ; j=1,2, \ldots, m\right\}$, where each element of the sequence can be expressed in terms of the linearly covariate error model with a constant standard deviation, see Linna and Woodall (2001). However, since this error model incorporate the AR(1) process with parameter $\phi^{s+1}$, then it written in a slightly different manner, i.e.,

$$
X_{t,(s+1) i-s, j}=A+B Y_{t,(s+1) i-s}+e_{t,(s+1) i-s, j}
$$

where $e_{t,(s+1) i-s, j} \sim N\left(0, \sigma_{M}\right)$ is a random error term due to measurement inaccuracy and $\sigma_{M}$ is the standard deviation of the measurement system. For the sake of simplicity, in this paper, it is assumed that $A=0$ and $B=1$, where $A$ and $B$ are two constants depending on the measurement system location error. At 
each sampling point, with $d_{r}$ sampling interval, there are $m$ separate measurements, each of size $n_{r}$ (i.e. a total of $m \times n_{r}$ observations), so that the plotting statistics are obtained as,

$$
\bar{X}_{t}=\frac{1}{m n_{r}} \sum_{i=1}^{n_{r}} \sum_{j=1}^{m} X_{t,(s+1) i-s, j}=\frac{1}{n_{r}}\left(\sum_{i=1}^{n_{r}} Y_{t,(s+1) i-s}+\frac{1}{m} \sum_{i=1}^{n_{r}} \sum_{j=1}^{m} e_{t,(s+1) i-s, j}\right) .
$$

Since $\bar{X}_{t}$ is assumed to be from an imperfect measurement system (i.e. $\gamma=\frac{\sigma_{M}}{\sigma_{0}}$, which denotes the ratio of the measurement system variability to the process variability, where $0 \leq \gamma \leq 1)$ then it is standard practice to take multiple measurements (i.e. $m$-measurement strategy, with $m>1$ ) as a remedial approach in reducing the negative effect of measurement errors, see for instance the review paper by Maleki et al. (2017). The standard deviation of the process in Equation (3) at every $d_{r}$ sampling interval is given by

with,

$$
\sigma_{\bar{X}_{t}}=\frac{\sigma_{0}}{\sqrt{n_{r}}} \rho\left(n_{r}, m, \gamma, s, \phi\right)
$$

$$
\rho\left(n_{r}, m, \gamma, s, \phi\right)=\sqrt{\left(\frac{m+\gamma^{2}}{m}\right)+\left(\frac{n_{r}+2\left(\frac{\phi^{(s+1)\left(n_{r}+1\right)}-n_{r} \phi^{2 s+2}+\left(n_{r}-1\right) \phi^{s+1}}{\left(\phi^{s+1}-1\right)^{2}}\right)}{n_{r}}\right)-1}
$$

Note that when $\phi=\gamma=0$ (i.e., the i.i.d. case), then $\rho\left(n_{r}, m, \gamma, s, \phi\right)$ is simply equal to 1 for any value of $m, s$ or $n_{r}$. The standardized charting statistic for each sample of size $n_{r}$ at the $t^{\text {th }}$ sampling point, at every $d_{r}$ sampling interval is given by

$$
Z_{t}=\frac{\bar{X}_{t}-\mu_{0}}{\sigma_{\bar{X}_{t}}}
$$

where $\bar{X}_{t} \sim N\left(\mu_{1}, \sigma_{\bar{X}_{t}}\right)$. When $\delta=0$, it means that the process is IC and $Z_{t} \sim N(0,1)$. However, when $\delta \neq$ 0 , the process is OOC and $Z_{t} \sim N(\delta, 1)$.

\section{Operation and run-length properties of the VSSI-Runs and VSSI-Synth schemes}

\subsection{Operation of the VSSI-Runs and VSSI-Synth schemes}

In order to decide when to take larger / smaller samples or wait short / long intervals before sampling, the area between the control limits is essentially divided into multiple regions by warning / control limits. Depending on the area the point is plotted, a decision is made concerning the values of $n_{r}$ and $d_{r}$ for the next sample. Hence, in monitoring the process mean, samples of size $n_{r}$ are usually taken at each sampling point $t$ after $d_{r}$ time interval to be inspected and then, each of these samples, are classified as either conforming or nonconforming depending on where the sample point plots on the charting regions shown in Figure 1; with limits given by: lower control limit $(L C L)$, lower outer warning limit $(L O W L)$, lower inner warning limit $(L I W L)$, center line $(C L)$, upper inner warning limit $(U I W L)$, upper outer warning limit $(U O W L)$ and upper control limit $(U C L)$. In Table 1, the operational procedure of each of the VSSI-Synth and VSSI-Runs monitoring schemes is presented, where the metric, average time to signal (ATS), is the average number of time periods until an OOC signal is generated by a monitoring scheme and $A T S_{0}$ denotes the desired nominal ATS. Note that $C R L_{L}\left(C R L_{U}\right)$ is the number of conforming samples that fall in region $\mathrm{E}^{-}\left(\mathrm{E}^{+}\right)$; which are in between the two consecutive nonconforming samples that fall on region $\mathrm{C}^{-}\left(\mathrm{C}^{+}\right)$, respectively. Moreover, each computation of the $C R L$ value includes the nonconforming sample at the end, so that the absence of any nonconforming sample means that $C R L_{L}$ or $C R L_{U}$ is equal to 1. 


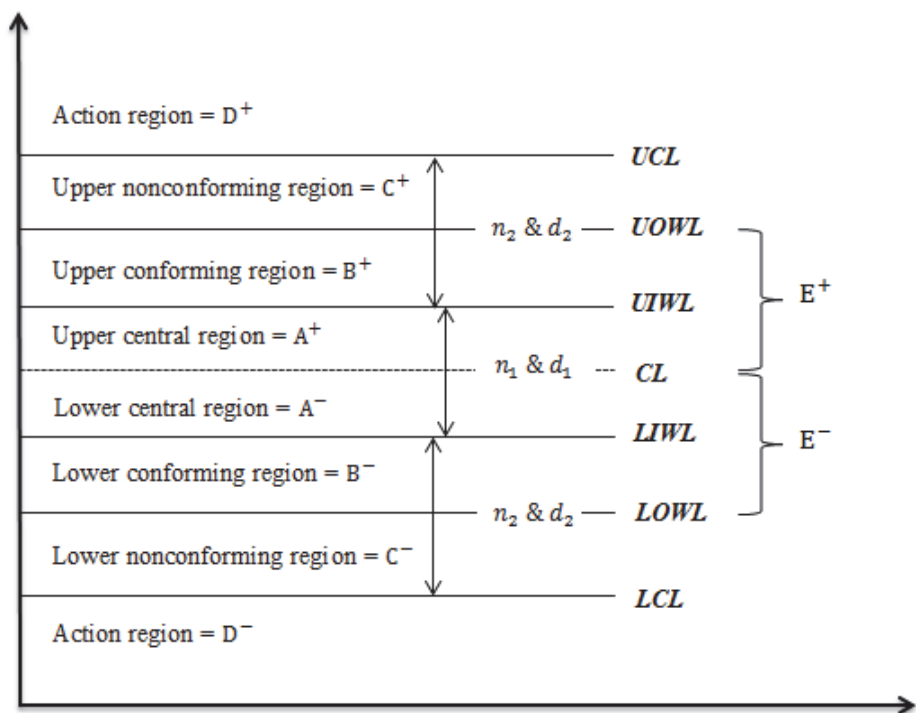

Fig. 1. The control and warning limits and the corresponding regions of the VSSI-Synth and VSSIRuns $\bar{X}$ sub-schemes

Table 1

Operation of the VSSI-Runs and VSSI-Synth $s \& m \bar{X}$ monitoring schemes

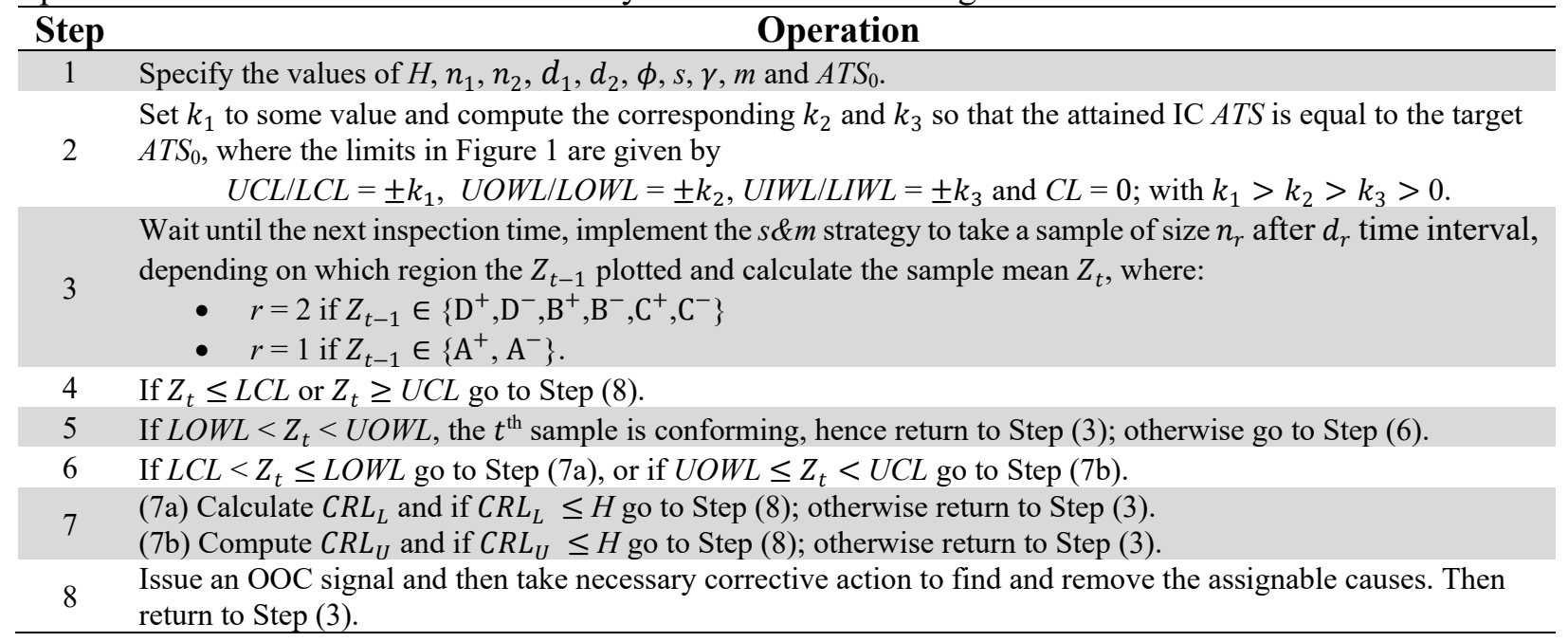

\subsection{Run-length properties using the Markov chain TPM}

The first sample that is taken from the process when it is starting or after an OOC signal is randomly decided to be of size $n_{1}$ and at sampling interval $d_{1}$ with probability $p_{0}$, or of size $n_{2}$ and at sampling interval $d_{2}$ with probability $1-p_{0}$, where $p_{0}=\Phi\left(k_{3}\right)-\Phi\left(-k_{3}\right)$. That is, $p_{0}$ corresponds to states with $\left(n_{1} \& d_{1}\right)$, whereas $\left(1-p_{0}\right)$ corresponds to states with $\left(n_{2} \& d_{2}\right)$, so that during the IC period, the rate of inspected items per sampling or average sample size (i.e., $\bar{n}$ ) is given by (see for instance, Costa and Machado, 2016)

$\bar{n}=n_{1} p_{0}+n_{2}\left(1-p_{0}\right)$,

whereas; the average sampling interval (i.e. $\bar{d}$ ) is given by

$\bar{d}=d_{1} p_{0}+d_{2}\left(1-p_{0}\right)$.

Jensen et al. (2008) citing a number of earlier works, stated that the use of the ATS as well as the standard deviation of the number of time periods to signal (SDTS) to measure the performance of a monitoring scheme is recommended for a VSSI approach. The latter metrics are respectively given by:

$A T S=\xi^{\mathrm{T}}(\mathbf{I}-\mathbf{Q})^{-1} \mathbf{d}$, 


$$
S D T S=\sqrt{\xi^{\mathrm{T}}(\mathbf{I}-\mathbf{Q})^{-1}\left(2 \mathbf{D}_{\mathbf{d}}(\mathbf{I}-\mathbf{Q})^{-1} \mathbf{d}-\mathbf{d}^{(2)}\right)-A T S^{2}},
$$

where $\mathbf{Q}$ is the $M \times M$ essential TPM discussed in the Appendix, $\xi$ is a $M \times 1$ vector with entries equal to either $p_{0}$ or $\left(1-p_{0}\right)$ depending on which state the entry of the vector corresponds with which entry on the TPM, i.e., $p_{0}$ corresponds to states with $\left(n_{1} \& d_{1}\right)$, whereas $\left(1-p_{0}\right)$ corresponds to states with $\left(n_{2}\right.$ $\left.\& d_{2}\right)$. Similarly, $\mathbf{d}$ is a $M \times 1$ vector with entries equal to either $d_{1}$ or $d_{2}$ depending on which state it corresponds with on the TPM, i.e., $d_{1}$ corresponds to states with $\left(n_{1} \& d_{1}\right)$, whereas $d_{2}$ corresponds to states with $\left(n_{2} \& d_{2}\right)$. Next, $\mathbf{d}^{(2)}$ contains the squares of the elements of $\mathbf{d}, \mathbf{D}_{\mathbf{d}}$ is a $M \times M$ diagonal matrix containing the elements of $\mathbf{d}$ as diagonals and zeros elsewhere; finally, $\mathbf{I}$ is a $M \times M$ identity matrix. Furthermore, Jensen et al. (2008) noted that to measure the number of switches from the state $\left(n_{1} \& d_{1}\right)$ to the state $\left(n_{2} \& d_{2}\right)$ and vice versa - to calculate these, the average number of switches to signal while the process is IC (denoted by $A N S W$ ) and its standard deviation (denoted by $S D N S W$ ) are calculated using:

$$
S D N S W=\sqrt{\xi^{\mathrm{T}}(\mathbf{I}-\mathbf{Q})^{-1}\left(2 \mathbf{D}_{\mathbf{p}}(\mathbf{I}-\mathbf{Q})^{-1} \mathbf{p}-\mathbf{p}^{(2)}\right)-A N S W^{2}},
$$

where $\mathbf{p}$ is a $M \times 1$ vector with entries equal to either $p_{1}$ or $p_{2}$; where $p_{1}$ is the probability of moving from state $\left(n_{1} \& d_{1}\right)$ to state $\left(n_{2} \& d_{2}\right)$, and $p_{2}$ is the probability of moving from state $\left(n_{2} \& d_{2}\right)$ to state $\left(n_{1}\right.$ $\left.\& d_{1}\right)$, which are both calculated while $\delta=0$. Moreover, $\mathbf{p}^{(2)}$ contains the squares of the elements of $\mathbf{p}$ and $\mathbf{D}_{\mathbf{p}}$ is a $M \times M$ diagonal matrix containing the elements of $\mathbf{p}$ as diagonals and zeros elsewhere. To evaluate the performance of the proposed schemes from an overall performance perspective, the expected $A T S$ (EATS) and the expected SDTS (ESDTS) are used because users tend not to know beforehand what exact shift value(s) is targeted - see for example, Khoo et al. (2019) and Mabude et al. (2020). The EATS and ESDTS measure the performance of a monitoring scheme over a range of shift values, i.e. $\delta_{\min }$ to $\delta_{\max }-$ which are the lower and the upper bound of $\delta$, respectively. Note that the shifts within the interval $\left[\delta_{\min }, \delta_{\max }\right]$ usually occur according to a pdf which is usually unknown. In the absence of any particular information, it is usually assumed that the shifts in the process mean happen with an equal probability, i.e., a Uniform $\left(\delta_{\min }, \delta_{\max }\right)$ distribution. The proposed scheme is designed such that, $k_{1}$ and $k_{3}$ are fixed, then implement a search algorithm for a value of $k_{2}$ so that the attained IC ATS is equal to the target $A T S_{0}$. Thus, the combination of design parameters that yields the best overall performance for a range of specified shifts is chosen and it is achieved by using $\left(H, k_{1}, k_{2}^{*}, k_{3}\right)=\underset{\left(H, k_{1}, k_{2}, k_{3}\right)}{\operatorname{argmin}} E A T S$, with

$$
E A T S=\frac{1}{\Delta} \sum_{\delta=\delta_{\min }}^{\delta_{\max }} \operatorname{ATS}(\delta) \text { and also, } \operatorname{ESDTS}=\frac{1}{\Delta} \sum_{\delta=\delta_{\min }}^{\delta_{\max }} \operatorname{SDTS}(\delta)
$$

subject to $\operatorname{ATS}(\delta=0)=A T S_{0}$, with $\delta \in\left[\delta_{\min }, \delta_{\max }\right]$ and $\Delta$ is the number of increments from $\delta_{\min }$ to $\delta_{\text {max }}$ of a Riemann sum. Moreover, $A T S(\delta)$ and $S D T S(\delta)$ are the ATS and SDTS as a function of the shift $\delta$ in the parameter under surveillance. However, the expected $A N S W$ (denoted by EANSW) and expected $S D N S W(E S D N S W)$ are given by

$$
E A N S W=\frac{1}{\Delta} \sum_{\delta=\delta_{\min }}^{\delta_{\max }} A N S W(\delta) \text { and } \operatorname{ESDNSW}=\frac{1}{\Delta} \sum_{\delta=\delta_{\min }}^{\delta_{\max }} \operatorname{SDNSW}(\delta)
$$

where $A N S W(\delta)$ and $S D N S W(\delta)$ are the $A N S W$ and $S D N S W$ as a function of $\delta$ in the parameter under surveillance. Finally, to investigate the effect of varying the value of $(\phi, \gamma)$ yields in terms of the monitoring scheme's performance at some specific values of $s \& m$, the percentage difference (\%Diff) is defined. For instance, the EATS's is calculated as, $\% \operatorname{Diff}_{\mathrm{A}}=\frac{\operatorname{EATS}_{(\phi, \gamma)}-\operatorname{EATS}_{(0,0)}}{\operatorname{EATS}_{(0,0)}} \times 100 \%$, where $\operatorname{EATS}_{(\phi, \gamma)}$ denote the EATS at some specific values of $\phi$ and $\gamma$, whereas $\operatorname{EATS}_{(0,0)}$ denotes the one of the 
i.i.d. case. The other \%Diff are defined in a similar manner, i.e., $\% \operatorname{Diff}_{\mathrm{SD}}=\frac{\operatorname{ESDTS}_{(\phi, \gamma)}-\operatorname{ESDTS}_{(0,0)}}{\operatorname{ESDTS}_{(0,0)}} \times 100 \%$, $\% \operatorname{Diff}_{\mathrm{AN}}=\frac{\operatorname{EANSW}_{(\phi, \gamma)}-\operatorname{EANSW}_{(0,0)}}{\operatorname{EANSW}_{(0,0)}} \times 100 \%, \quad \% \operatorname{Diff}_{\mathrm{SDN}}=\frac{\operatorname{ESDNSW}_{(\phi, \gamma)}-\operatorname{ESDNSW}_{(0,0)}}{\operatorname{ESDNSW}_{(0,0)}} \times 100 \%$ for the ESDTS, EANSW and ESDNSW, respectively.

\section{Empirical analysis of the VSSI approach for the proposed monitoring schemes}

\subsection{IC design considerations}

Since the design parameters (i.e. $H, k_{1}, k_{2}$ and $k_{3}$ ) are determined while the process is in a state of IC, then they are the same as those for i.i.d. observations because they do not depend on the level of autocorrelation and / or measurement errors. Note though, the VSSI-Synth and VSSI-Runs monitoring schemes for i.i.d. observations do not exist in the SPM literature, hence, they will also be discussed here. Note that, for i.i.d. observations, Yu et al. (2016) investigated the VSSI synthetic schemes using the NSS design with fewer charting regions so that it has two design parameters $\left(k_{1}\right.$ and $\left.k_{2}\right)$ for a given $H$, instead of three as discussed here. For illustration purpose, the design parameters are only shown for $k_{1} \in\{3.1$, $3.5,4.0\}$ and $k_{3}=0.6724$, separately, in the zero- and steady-state modes for $A T S_{0}=370.4$ in Table 2 . Based on the boldfaced values, it is observed that as $H$ increases, the values of $k_{2}$ such that the attained IC $A T S$ is equal to $A T S_{0}$ converge to some specific value no matter how large $H$ is. A similar pattern in $k_{2}$ values is observed for other different values of $k_{1}$ and $k_{3}$ as well as at different desired values of $A T S_{0}$. In the zero-state mode, the VSSI-Runs and VSSI-Synth monitoring schemes have different $k_{2}$ design parameters (for some given $k_{1}$ and $k_{3}$ ); however, in the steady-state mode, these two schemes are equivalent, and thus the $k_{2}$ design parameters are the same - see a thorough discussion in the Appendix.

Table 2

The values of $k_{2}$ for the zero- and steady-state VSSI-Runs and VSSI-Synth schemes when $k_{1} \in\{3.1,3.5$, $4.0\}, H \in\{1,2, \ldots, 20\}, A T S_{0}=370.4$ and $k_{3}=0.6724$

\begin{tabular}{|c|c|c|c|c|c|c|c|c|c|}
\hline & \multicolumn{3}{|c|}{$k_{1}=3.1$} & \multicolumn{3}{|c|}{$k_{1}=3.5$} & \multicolumn{3}{|c|}{$k_{1}=4.0$} \\
\hline & Zero-state & Zero-state & Steady-state & Zero-state & Zero-state & Steady-state & $\begin{array}{c}\text { Zero- } \\
\text { state }\end{array}$ & Zero-state & Steady-state \\
\hline$H$ & Runs & Synth & Runs\&Synth & Runs & Synth & Runs\&Synth & Runs & Synth & Runs\&Synth \\
\hline 1 & 2.0393 & 2.0664 & 2.0398 & 1.8221 & 1.8401 & 1.8227 & 1.7866 & 1.8035 & 1.7872 \\
\hline 2 & 2.1150 & 2.1469 & 2.1157 & 1.9056 & 1.9269 & 1.9064 & 1.8713 & 1.8914 & 1.8721 \\
\hline 3 & 2.1425 & 2.1765 & 2.1433 & 1.9357 & 1.9585 & 1.9366 & 1.9018 & 1.9232 & 1.9027 \\
\hline 4 & 2.1544 & 2.1894 & 2.1553 & 1.9486 & 1.9721 & 1.9496 & 1.9148 & 1.9369 & 1.9158 \\
\hline 5 & 2.1600 & 2.1954 & 2.1609 & 1.9545 & 1.9782 & 1.9555 & 1.9207 & 1.9431 & 1.9218 \\
\hline 6 & 2.1626 & 2.1982 & 2.1635 & 1.9572 & 1.9811 & 1.9583 & 1.9235 & 1.9460 & 1.9246 \\
\hline 7 & 2.1638 & 2.1996 & 2.1648 & 1.9585 & 1.9825 & 1.9596 & 1.9248 & 1.9473 & 1.9259 \\
\hline 8 & 2.1644 & 2.2002 & 2.1654 & 1.9591 & 1.9832 & 1.9602 & 1.9254 & 1.9480 & 1.9265 \\
\hline 9 & 2.1647 & 2.2006 & 2.1657 & 1.9594 & 1.9835 & 1.9605 & 1.9257 & 1.9483 & 1.9268 \\
\hline 10 & 2.1649 & 2.2007 & 2.1659 & 1.9595 & 1.9836 & 1.9606 & 1.9258 & 1.9484 & 1.9269 \\
\hline 11 & 2.1649 & 2.2008 & 2.1659 & 1.9596 & 1.9837 & 1.9607 & 1.9259 & 1.9485 & 1.9270 \\
\hline 12 & 2.1650 & 2.2008 & 2.1660 & 1.9597 & 1.9837 & 1.9608 & 1.9259 & 1.9485 & 1.9270 \\
\hline 13 & 2.1650 & 2.2009 & 2.1660 & 1.9597 & 1.9837 & 1.9608 & 1.9259 & 1.9485 & 1.9270 \\
\hline 14 & 2.1650 & 2.2009 & 2.1660 & 1.9597 & 1.9837 & 1.9608 & 1.9259 & 1.9486 & 1.9270 \\
\hline 15 & 2.1650 & 2.2009 & 2.1660 & 1.9597 & 1.9837 & 1.9608 & 1.9259 & 1.9486 & 1.9270 \\
\hline 16 & 2.1650 & 2.2009 & 2.1660 & 1.9597 & 1.9837 & 1.9608 & 1.9259 & 1.9486 & 1.9270 \\
\hline 17 & 2.1650 & 2.2009 & 2.1660 & 1.9597 & 1.9837 & 1.9608 & 1.9259 & 1.9486 & 1.9270 \\
\hline 18 & 2.1650 & 2.2009 & 2.1660 & 1.9597 & 1.9837 & 1.9608 & 1.9259 & 1.9486 & 1.9270 \\
\hline 19 & 2.1650 & 2.2009 & 2.1660 & 1.9597 & 1.9837 & 1.9608 & 1.9259 & 1.9486 & 1.9270 \\
\hline 20 & 2.1650 & 2.2009 & 2.1660 & 1.9597 & 1.9837 & 1.9608 & 1.9259 & 1.9486 & 1.9270 \\
\hline$\vdots$ & $\vdots$ & $\vdots$ & $\vdots$ & $\vdots$ & $\vdots$ & $\vdots$ & $\vdots$ & $\vdots$ & $\vdots$ \\
\hline 100 & 2.1650 & 2.2009 & 2.1660 & 1.9597 & 1.9837 & 1.9608 & 1.9259 & 1.9486 & 1.9270 \\
\hline$\vdots$ & $\vdots$ & $\vdots$ & $\vdots$ & $\vdots$ & $\vdots$ & $\vdots$ & $\vdots$ & $\vdots$ & $\vdots$ \\
\hline
\end{tabular}




\subsection{OOC performance}

In Tables 3 and 4, the run-length properties (i.e. ATS, SDTS, ANSW, SDNSW, EATS, ESDTS, EANSW and ESDNSW) from Eq. (9) to Eq. (12) are used to evaluate the proposed VSSI-Synth and VSSI-Runs monitoring schemes in zero- and steady-state modes and then compare their OOC performance with the Costa and Castagliola (2011)'s FSSI $\bar{X}$ scheme as well as the VSSI $\bar{X}$ scheme when:

(i) $\phi=\gamma=0$, i.e. the i.i.d. case,

(ii) $\phi=\gamma=0.75$, i.e. the combined effect of autocorrelation and measurement errors.

Firstly, for the i.i.d. case, it is observed from Panel (a) of Table 3 that in the zero-state mode, the VSSISynth monitoring scheme has the best OOC ATS at all considered shift values and consequently, the lowest EATS. However, the corresponding SDTS is slightly higher when the process is IC and other small shift values; hence, the corresponding ESDTS is higher than that of the VSSI-Synth scheme in steadystate and VSSI-Runs scheme in zero-state.

Similarly, in Panel (a) of Table 4, the OOC ANSW and EANSW of the VSSI-Synth scheme in zero-state is the smallest, the corresponding ESDNSW are higher. Since the sample size and sampling interval are fixed at $\bar{n}$ and $\bar{d}$ for the FSSI $\bar{X}$ monitoring scheme, there are no switches; hence, it is not considered in Table 4. Secondly, the OOC performance of the VSSI-Runs scheme in steady-state mode is approximately equal to that of the VSSI-Runs scheme in zero-state mode. Consequently, the resulting deductions are summarized in Remark 1.

Remark 1: Let the zero- and steady-state EATS be denoted by ZSEATS and SSEATS, respectively. Then the percentage difference for the VSSI-Runs scheme (calculated using $\left|\frac{\text { ZSEATS-SSEATS }}{\text { SSEATS }}\right| \times 100 \%$ ) are always less than $1 \%$. Similarly, the same deduction is observed for the zero- and steady-state ESDTS, $E A N S W$ and $E S D N S W$ of the VSSI-Runs scheme. As it can be seen from Tables 3 and 4, similar results can be observed for the processes under the combined effect of autocorrelation and measurement errors. In order to preserve writing space, the VSSI-Runs scheme in the steady-state mode only is considered hereafter.

Thirdly, when the process is under the combined effect of autocorrelation and measurement errors, all the corresponding run-length properties are inflated as the latter two factors have a negative effect towards the OOC performance. In Panel (b) of Tables 3 and 4, the 'no remedy strategy' denotes a scenario where $s=0$ (i.e. no skip) and $m=1$ (i.e. single measurement). Hence, based on the '\%Diff' values, it is observed that for each corresponding scheme there is deterioration in performance as all the percentage values are large.

Fourthly, implementing the $s \& m$ strategy (i.e. $s=3 \& m=4$ ), reduces the combined negative effect of autocorrelation and measurement errors as compared to the no remedy strategy because all the \%Diff values are slightly closer to those of the i.i.d. case for all the considered schemes in Tables 3 and 4.

Overall, it is observed that at each instance, the proposed schemes have a better OOC performance than the Costa and Castagliola (2011)'s FSSI $\bar{X}$ scheme as well as the corresponding VSSI $\bar{X}$ scheme. Although the VSSI-Synth scheme has the best ATS and ANSW performance in zero-state, their corresponding variability are higher than those in steady-state mode. Due to the lower variability in the steady-state mode and taking into account Remark 1, moving forth, the focus is on the VSSI-Synth / VSSI-Runs scheme in the steady-state mode. 


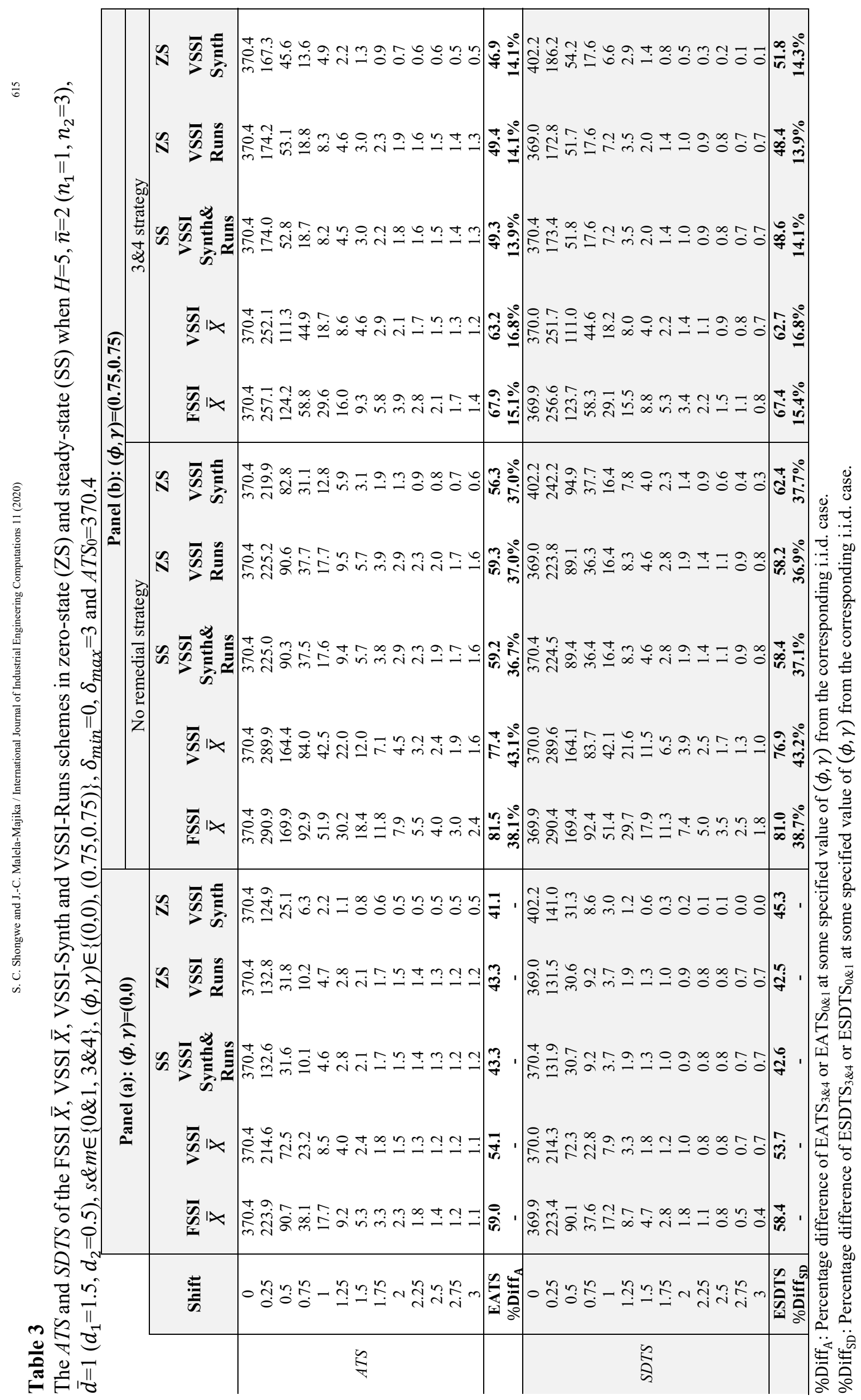




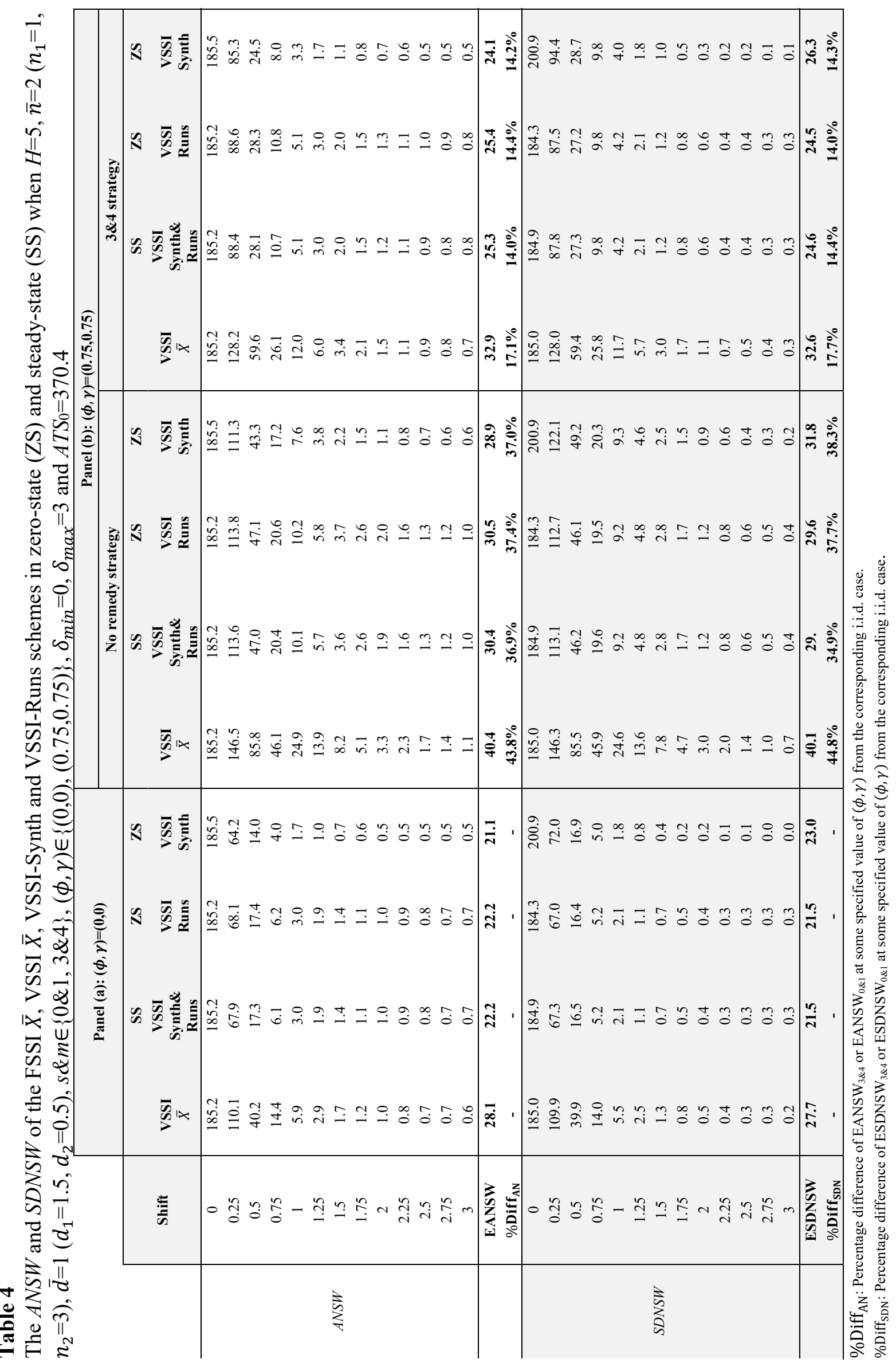


Next, in Table 5, the effect of varying the sample size and sampling interval on the performance of the FSSI $\bar{X}$, VSSI $\bar{X}$, VSSI-Synth and VSSI-Runs schemes is studied. Firstly, for all the schemes, using the $3 \& 4$ strategy instead of the no remedy strategy yields better EATS for different values of $\phi>0$ and $\gamma>0$. Secondly, for each pair $(\phi, \gamma)$, increasing $\bar{n}$ leads to an improved performance for all the considered schemes. Thirdly, for each pair $(\phi, \gamma)$, when decreasing $d_{1}$ (while increasing $d_{2}$ so that $\bar{d}$ remains constant) leads to an improved performance for all the considered schemes; however, the converse leads to a deterioration, i.e. increasing $d_{1}$ (while decreasing $d_{2}$ so that $\bar{d}$ remains constant). Lastly, at any specific combination: $\phi, \gamma, \bar{n}$ and $\bar{d}$, the proposed VSSI-Synth / VSSI-Runs scheme has the best OOC performance than the competing FSSI and VSSI $\bar{X}$ schemes.

\section{Table 5}

The EATS of the FSSI and VSSI $\bar{X}$ schemes as well as steady-state VSSI-Synth and VSSI-Runs schemes when using a no remedy approach (and $3 \& 4$ - in brackets) strategies with $H=5, \bar{d}=1$, $\delta_{\min }=0, \delta_{\max }=3, A T S_{0}=370.4$ and $\bar{n} \in\{2,5,7\}$

\begin{tabular}{ccccccc} 
& & \multirow{2}{*}{ FSSI $\bar{X}$} & \multicolumn{2}{c}{ VSSI $\bar{X}$} & \multicolumn{2}{c}{ VSSI-Synth \& VSSI-Runs } \\
$(\phi, \gamma)$ & $\bar{n}$ & & $d_{1}=0.5, d_{2}=1.5$ & $d_{1}=0.01, d_{2}=1.99$ & $d_{1}=0.5, d_{2}=1.5$ & $d_{1}=0.01, d_{2}=1.99$ \\
\hline \multirow{3}{*}{$(0.25,0.25)$} & 2 & $65.3(59.4)$ & $60.5(54.5)$ & $58.1(52.5)$ & $47.5(43.5)$ & $46.4(42.5)$ \\
& 5 & $49.8(43.6)$ & $45.8(40.2)$ & $44.3(38.9)$ & $37.9(34.6)$ & $37.1(34.0)$ \\
& 7 & $45.1(39.6)$ & $42.1(37.2)$ & $40.7(36.1)$ & $35.6(32.9)$ & $35.0(32.4)$ \\
\hline \multirow{3}{*}{$(0.5,0.5)$} & 2 & $73.0(61.6)$ & $68.4(56.6)$ & $65.5(54.5)$ & $53.0(44.9)$ & $51.6(43.9)$ \\
& 5 & $58.7(45.4)$ & $54.2(41.8)$ & $52.1(40.5)$ & $43.3(35.6)$ & $42.2(34.9)$ \\
\hline & 7 & $53.6(41.1)$ & $49.9(38.5)$ & $48.0(37.3)$ & $40.4(33.7)$ & $39.5(33.1)$ \\
\hline & 2 & $81.5(67.9)$ & $77.4(63.2)$ & $74.0(60.6)$ & $59.2(49.3)$ & $57.7(48.1)$ \\
& 5 & $69.8(52.4)$ & $65.4(48.2)$ & $62.6(46.5)$ & $50.8(39.4)$ & $49.5(38.5)$ \\
\hline
\end{tabular}

NB: $\bar{n}$ equal to 2,5 and 7 implies that $\left(n_{1}=1, n_{2}=3\right),\left(n_{1}=2, n_{2}=8\right)$ and $\left(n_{1}=4, n_{2}=10\right)$, respectively

Next, in Tables 6 and 7, the EATS, ESDTS, EANSW and ESDNSW are given for the VSSI-Synth / VSSIRuns scheme in steady-state when $\phi=\gamma=0.5$ (in Table 7) and those of the i.i.d. case (in Table 6) with $\bar{n}$ equal to 5 and 7 . The '\%Diff' in brackets in Table 7 are computed using the corresponding i.i.d. EATS, ESDTS, EANSW and ESDNSW values in Table 6. From Table 6, as well as for each $s \& m$ in Table 7, it is observed that, while the EATS and ESDTS decrease as $d_{1}$ decreases (or $d_{2}$ increases) for a specific $\bar{n}$; however, the corresponding EANSW and ESDNSW remain constant. For each $\left(d_{1}, d_{2}\right)$ and / or $\bar{n}$ : An improvement in performance with the largest desired effect occurs when both $s$ and $m$ are increased. For example, for $\left(d_{1}, d_{2}\right)=(0.5,1.5)$ and $\bar{n}=5$, the $E A T S=43.3$ and $\%$ Diff $_{\mathrm{A}}=25.5 \%$ when $s=0$ and $m=1$ in Table 7 , hence increasing both $s$ and $m$ to 1 and 2 yields $E A T S=38.3$ and $\%$ Diff $_{\mathrm{A}}=11.0 \%$. Increasing either $s$ or $m$ yields the best improvement when $s$ is increased (with $m$ constant) as compared to the converse. For instance, for $\left(d_{1}, d_{2}\right)=(0.5,1.5)$ and $\bar{n}=5$, the $\% \operatorname{Diff}_{\mathrm{A}}=25.5 \%$ when $s=0$ and $m=1$ in Table 7 , hence increasing $s$ to 1 (with $m=1$ ) yields $\%$ Diff $_{\mathrm{A}}=13.0 \%$; however, increasing $m$ to 2 (with $s=0$ ) yields $\%$ Diff $_{\mathrm{A}}=23.5 \%$. Thus, it follows that, individually, the $s$-skip strategy has a greater improvement effect on the OOC performance than the multiple measurements strategy.

\section{Table 6}

The EATS (first row), ESDTS (second row), EANSW (third row) and ESDNSW (fourth row) for the steadystate VSSI-Synth and VSSI-Runs $\bar{X}$ scheme when $H=5, \bar{n} \in\{5,7\}, \bar{d}=1, \phi=\gamma=0$ and $A T S_{0}=370.4$

\begin{tabular}{|c|c|c|c|}
\hline \multirow{4}{*}{$\bar{n}=5$} & 34.5 & 34.0 & 33.9 \\
\hline & 33.9 & 34.0 & 34.0 \\
\hline & 17.6 & 17.6 & 17.6 \\
\hline & 17.0 & 17.0 & 17.0 \\
\hline \multirow{4}{*}{$\bar{n}=7$} & 32.8 & 32.4 & 32.3 \\
\hline & 32.3 & 32.4 & 32.4 \\
\hline & 16.7 & 16.7 & 16.7 \\
\hline & 16.2 & 16.2 & 16.2 \\
\hline$\left(d_{1}, d_{2}\right)$ & $(0.5,1.5)$ & $(0.1,1.9)$ & $(0.01,1.99)$ \\
\hline
\end{tabular}


Table 7

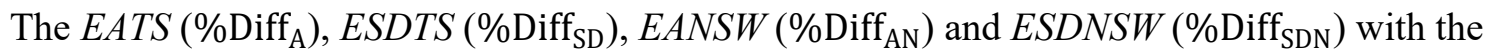
corresponding percentage difference (in brackets) from the i.i.d. case values for the steady-state VSSI-Synth and VSSI-Runs $\bar{X}$ scheme when $H=5, \bar{n} \in\{5,7\}, \bar{d}=1, \phi=\gamma=0.5$ and $A T S_{0}=370.4$

\begin{tabular}{|c|c|c|c|c|c|c|c|}
\hline & & & $s=0$ & & & $s=1$ & \\
\hline \multirow{7}{*}{$\bar{n}=5$} & \multirow{4}{*}{$m=1$} & $43.3(25.5 \%)$ & $42.4(24.7 \%)$ & $42.2(24.5 \%)$ & $39.0(13.0 \%)$ & $38.3(12.6 \%)$ & $38.2(12.7 \%)$ \\
\hline & & $42.6(25.7 \%)$ & $42.4(25.1 \%)$ & $42.4(25.1 \%)$ & $38.4(13.3 \%)$ & $38.3(12.6 \%)$ & $38.3(12.6 \%)$ \\
\hline & & $22.1(25.6 \%)$ & $22.1(25.6 \%)$ & $22.1(25.6 \%)$ & $19.9(13.1 \%)$ & $19.9(13.1 \%)$ & $19.9(13.1 \%)$ \\
\hline & & $21.5(26.5 \%)$ & $21.5(26.5 \%)$ & $21.5(26.5 \%)$ & $19.3(13.5 \%)$ & $19.3(13.5 \%)$ & $19.3(13.5 \%)$ \\
\hline & \multirow{4}{*}{$m=2$} & $42.6(23.5 \%)$ & $41.8(22.9 \%)$ & $41.6(22.7 \%)$ & $38.3(11.0 \%)$ & $37.6(10.6 \%)$ & $37.5(10.6 \%)$ \\
\hline & & $41.9(23.6 \%)$ & $41.8(22.9 \%)$ & $41.7(22.6 \%)$ & $37.7(11.2 \%)$ & $37.6(10.6 \%)$ & $37.6(10.6 \%)$ \\
\hline & & $21.8(23.9 \%)$ & $21.8(23.9 \%)$ & $21.8(23.9 \%)$ & $19.6(11.4 \%)$ & $19.6(11.4 \%)$ & $19.6(11.4 \%)$ \\
\hline \multirow{10}{*}{$\bar{n}=7$} & & $21.2(24.7 \%)$ & $21.2(24.7 \%)$ & $21.2(24.7 \%)$ & $19.0(11.8 \%)$ & $19.0(11.8 \%)$ & $19.0(11.8 \%)$ \\
\hline & \multirow{4}{*}{$m=1$} & $40.4(23.2 \%)$ & $39.7(22.5 \%)$ & $39.5(22.3 \%)$ & $36.5(11.3 \%)$ & $35.9(10.8 \%)$ & $35.7(10.5 \%)$ \\
\hline & & $39.7(22.9 \%)$ & $39.6(22.2 \%)$ & $39.6(22.2 \%)$ & $35.9(11.1 \%)$ & $35.8(10.5 \%)$ & $35.8(10.5 \%)$ \\
\hline & & $20.7(24.0 \%)$ & $20.7(24.0 \%)$ & $20.7(24.0 \%)$ & $18.6(11.4 \%)$ & $18.6(11.4 \%)$ & $18.6(11.4 \%)$ \\
\hline & & $20.1(24.1 \%)$ & $20.1(24.1 \%)$ & $20.1(24.1 \%)$ & $18.1(11.7 \%)$ & $18.1(11.7 \%)$ & $18.1(11.7 \%)$ \\
\hline & \multirow{4}{*}{$m=2$} & $39.9(21.6 \%)$ & $39.2(21.0 \%)$ & $39.0(20.7 \%)$ & $35.9(9.5 \%)$ & $35.3(9.0 \%)$ & $35.2(9.0 \%)$ \\
\hline & & $39.2(21.4 \%)$ & $39.1(20.7 \%)$ & $39.1(20.7 \%)$ & $35.3(9.3 \%)$ & $35.3(9.0 \%)$ & $35.3(9.0 \%)$ \\
\hline & & $20.4(22.2 \%)$ & $20.4(22.2 \%)$ & $20.4(22.2 \%)$ & $18.3(9.6 \%)$ & $18.3(9.6 \%)$ & $18.3(9.6 \%)$ \\
\hline & & $19.8(22.2 \%)$ & $19.8(22.2 \%)$ & $19.8(22.2 \%)$ & $17.8(9.9 \%)$ & $17.8(9.9 \%)$ & $17.8(9.9 \%)$ \\
\hline & $\left(d_{1}, d_{2}\right)$ & $(0.5,1.5)$ & $(0.1,1.9)$ & $(0.01,1.99)$ & $(0.5,1.5)$ & $(0.1,1.9)$ & $(0.01,1.99)$ \\
\hline
\end{tabular}

When the users of the proposed monitoring schemes are interested in small shift values, the values of $H$ that are at least 5 are recommended; also by keeping in mind the convergence property in Table 2 . However, for moderate to large shifts, $H$ values that are less than 5 are recommended. In order to avoid violating the concept of R\&R (repeatability and reproducibility) and rational subgroups, large values of $s$ and $m$ are not advised; see for instance, Linna and Woodall (2001), Costa and Castagliola (2011) and Maleki et al. (2017). Moreover, note that for a specific $n_{r}$, when $s$ or $m$ increase, the $\rho\left(n_{r}, m, \gamma, s, \phi\right)$ (see Equation (5)) decreases towards a value of 1 (i.e., a desired effect because this decreases the variability of the process under the combined effect of autocorrelation and measurement errors). Thus, the higher the values of $s$ and $m$, the closer the value of $\rho\left(n_{r}, m, \gamma, s, \phi\right)$ gets towards the value of 1 . Next, to obtain an OOC performance that is within $5 \%$ of the i.i.d. OOC performance in terms of the EATS (i.e. $\% \operatorname{Diff}_{\mathrm{A}}$ ), recommendations are as follows:

- For $\phi$ within the intervals $(0,0.3],(0.3,0.5],(0.5,0.6]$ and $(0.6,0.8]$, a value of $s$ equal to 1,2 , 3-4 and 5 is recommended, respectively; for any given value of $\gamma$.

- For $\gamma$ within the intervals $(0,0.4],(0.4,0.7]$ and $(0.7,1]$, a value of $m$ equal to 2,3 and 4 is recommended, respectively; for any given value of $\phi$.

In a 'big data' environment, much greater values of $s$ are required to ensure that the underlying autocorrelation is theoretically removed. Moreover, large values of $s$ are mandatory in the scenarios where $\phi>0.8$ to ensure that the OOC performance of the process under the combined effect of autocorrelation and measurement errors have a $\%$ Diff $_{A}$ within 5\%. Since measurement errors do not have as much of a greater negative effect on the OOC performance as compared to autocorrelation; hence, multiple measurements with a value of $m$ greater than 4 are not recommended.

\section{Implementation example}

An illustration example showing how to implement the $s \& m=1 \& 2$ strategy for the VSSI-Synth / VSSIRuns monitoring scheme and form rational subgroups of size $n_{1}=1$ and $n_{2}=3$, at sampling intervals $d_{1}=1.5$ hours and $d_{2}=0.5$ hours, assuming that the weight of the yogurt cup filling process given in Table 8 (taken from Costa and Castagliola (2011, page 670)) has been IC for a long time, i.e. a steady-state mode. The Phase I analysis of this process indicated that the weight of a yogurt cup, $X_{t, i, j}$, fits an $\operatorname{AR}(1)$ 
model with parameter $\phi=0.38$, an IC mean estimate, $\mu_{0}=124.9 \mathrm{~g}$, an IC standard deviation, $\sigma_{0}=0.76 \mathrm{~g}$ and the R\&R historical study indicates that $\sigma_{M}=0.24 \mathrm{~g}$, so that $\gamma=0.316$.

\section{Table 8}

The weight of the yogurt filling cup process dataset

\begin{tabular}{ccccccccccc}
\hline Sample number $(\boldsymbol{t})$ & $\boldsymbol{X}_{\boldsymbol{t , 1 , 1}}$ & $\boldsymbol{X}_{\boldsymbol{t , 1} \mathbf{2}}$ & $\boldsymbol{X}_{\boldsymbol{t}, \mathbf{2}, \mathbf{1}}$ & $\boldsymbol{X}_{\boldsymbol{t}, \mathbf{2}, \mathbf{2}}$ & $\boldsymbol{X}_{\boldsymbol{t}, \mathbf{3}, \mathbf{1}}$ & $\boldsymbol{X}_{\boldsymbol{t}, \mathbf{3}, \mathbf{2}}$ & $\boldsymbol{X}_{\boldsymbol{t}, \mathbf{4}, \mathbf{1}}$ & $\boldsymbol{X}_{\boldsymbol{t}, \mathbf{4}, \mathbf{2}}$ & $\boldsymbol{X}_{\boldsymbol{t}, \mathbf{5}, \mathbf{1}}$ & $\boldsymbol{X}_{\boldsymbol{t}, \mathbf{5}, \mathbf{2}}$ \\
\hline 1 & 124.9 & 124.8 & 125.9 & 125.9 & 125.2 & 124.8 & 124.6 & 124.1 & 124.8 & 124.4 \\
2 & 124.9 & 125.2 & 125.5 & 125.0 & 124.1 & 123.9 & 125.2 & 125.2 & 125.0 & 125.6 \\
3 & 125.1 & 125.1 & 125.2 & 124.8 & 125.4 & 125.3 & 122.9 & 122.4 & 125.4 & 125.4 \\
4 & 126.1 & 125.9 & 124.6 & 124.8 & 125.7 & 125.5 & 126.4 & 126.5 & 124.9 & 125.7 \\
5 & 125.8 & 125.7 & 122.6 & 122.6 & 124.1 & 123.5 & 126.1 & 126.3 & 124.9 & 125.0 \\
6 & 125.0 & 125.2 & 125.5 & 124.8 & 124.8 & 125.0 & 124.9 & 124.8 & 124.8 & 124.2 \\
7 & 124.2 & 124.6 & 125.8 & 125.3 & 125.4 & 125.5 & 126.4 & 126.2 & 125.1 & 125.2 \\
8 & 124.9 & 124.9 & 123.8 & 123.2 & 125.1 & 125.3 & 124.0 & 124.5 & 124.4 & 124.2 \\
9 & 125.9 & 125.8 & 124.4 & 124.8 & 126.3 & 125.7 & 124.9 & 125.2 & 125.2 & 125.1 \\
10 & 124.2 & 124.3 & 126.2 & 125.5 & 125.6 & 125.0 & 124.4 & 124.4 & 124.1 & 124.3 \\
11 & 123.7 & 123.6 & 123.4 & 123.3 & 124.7 & 124.8 & 123.1 & 123.1 & 123.1 & 122.8 \\
12 & 124.0 & 124.1 & 122.6 & 122.4 & 123.6 & 123.6 & 124.4 & 124.5 & 123.6 & 123.1 \\
13 & 122.0 & 122.5 & 123.9 & 124.0 & 123.7 & 124.1 & 124.3 & 124.4 & 121.9 & 122.9 \\
14 & 122.4 & 123.0 & 122.8 & 123.1 & 123.7 & 124.2 & 123.7 & 124.1 & 122.8 & 123.1 \\
15 & 123.9 & 123.6 & 124.1 & 124.5 & 123.4 & 122.9 & 123.1 & 123.1 & 124.5 & 125.1 \\
16 & 121.9 & 122.3 & 123.4 & 123.3 & 123.5 & 123.3 & 125.3 & 125.5 & 123.3 & 123.6 \\
17 & 123.3 & 122.9 & 123.6 & 123.5 & 124.2 & 123.8 & 123.4 & 123.6 & 123.5 & 123.4 \\
18 & 122.0 & 122.2 & 123.6 & 123.4 & 124.7 & 125.0 & 122.6 & 122.5 & 124.5 & 123.9 \\
19 & 124.0 & 123.9 & 123.1 & 123.4 & 123.9 & 124.5 & 122.6 & 122.8 & 124.2 & 123.5 \\
20 & 125.5 & 124.9 & 122.2 & 122.3 & 123.2 & 123.2 & 123.2 & 123.3 & 123.2 & 123.2 \\
\hline
\end{tabular}

For illustration purpose, assume $H=1, k_{1}=3.5$ and $A T S_{0}=370.4$, then from Table 2 , it follows that $k_{2}=1.8227$ and $k_{3}=0.6724$; see Steps 1 and 2 in Table 1. Using Equation (4), $\rho\left(n_{r}, m, \gamma, s, \phi\right)$ and $\sigma_{\bar{X}_{t}}$ are equal to 1.0247 and 0.7787 when $n_{1}=1$, but are equal to 1.1209 and 0.4918 when $n_{2}=3$. For example, the charting statistics of the sample numbers 9 and 10 are calculated as follows:

- At sample number 9, the sample size \& interval are equal to $n_{1} \& d_{1}$ as $Z_{8}$ plotted in region $\mathrm{A}^{+}$(see Step (3) in Table 1) and the cumulative time is equal to 12.5 ; hence,

$\bar{X}_{9}=\frac{1}{m \times n_{1}}\left(X_{9,1,1}+X_{9,1,2}\right)=125.85$ and $Z_{9}=\frac{125.85-124.9}{0.7787}=1.22$, plotting in region $\mathrm{B}^{+}$.

- Since $Z_{9}$ plots in Region $\mathrm{B}^{+}$, then the sample size $\&$ interval at sample number 10 are equal to $n_{2} \& d_{2}$, and the cumulative time is equal to 13 ; and

$\bar{X}_{10}=\frac{1}{m \times n_{2}}\left(X_{10,1,1}+X_{10,1,2}+X_{10,3,1}+X_{10,3,2}+X_{10,5,1}+X_{10,5,2}\right)=124.58$ and $Z_{10}=\frac{126.00-124.58}{0.7787}=-0.64$,

falling in region $\mathrm{A}^{-}$; hence, at the next sampling point, the sample size $\&$ interval are equal to $n_{1} \& d_{1}$.

The rest of the charting procedure is done in a similar manner.

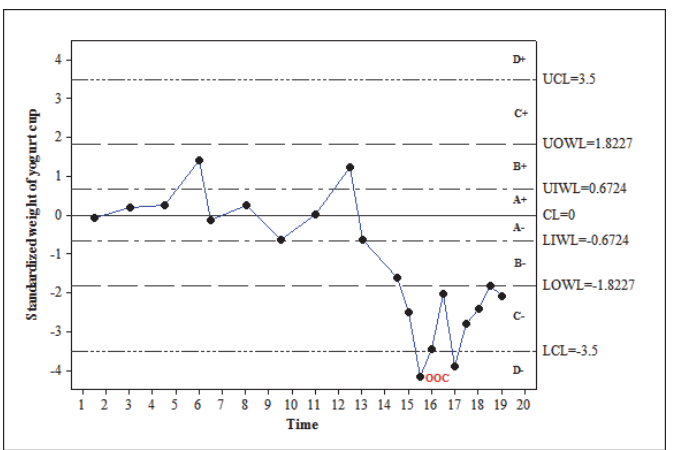

Fig. 2. Monitoring the weight of the yogurt cups using the VSSI-Synth / VSSI-Runs scheme with the $1 \& 2$ sampling strategy 
Table 9

The charting statistics of the VSSI-Synth / VSSI-Runs monitoring scheme using the $1 \& 2$ sampling strategy

\begin{tabular}{cccccc}
\hline $\begin{array}{c}\text { Sample } \\
\text { number }\end{array}$ & $\left(n_{r}, d_{r}\right)$ & Cumulative time & $\bar{X}_{t}$ & $Z_{t}$ & Region \\
\hline 1 & $\left(n_{1}, d_{1}\right)$ & 1.5 & 124.85 & -0.06 & $\mathrm{~A}^{-}$ \\
2 & $\left(n_{1}, d_{1}\right)$ & 3 & 125.05 & 0.19 & $\mathrm{~A}^{+}$ \\
3 & $\left(n_{1}, d_{1}\right)$ & 4.5 & 125.10 & 0.26 & $\mathrm{~A}^{+}$ \\
4 & $\left(n_{1}, d_{1}\right)$ & 6 & 126.00 & 1.41 & $\mathrm{~B}^{+}$ \\
5 & $\left(n_{2}, d_{2}\right)$ & 6.5 & 124.83 & -0.14 & $\mathrm{~A}^{-}$ \\
6 & $\left(n_{1}, d_{1}\right)$ & 8 & 125.10 & 0.26 & $\mathrm{~A}^{+}$ \\
7 & $\left(n_{1}, d_{1}\right)$ & 9.5 & 124.40 & -0.64 & $\mathrm{~A}^{-}$ \\
8 & $\left(n_{1}, d_{1}\right)$ & 11 & 124.90 & 0.00 & $\mathrm{~A}^{+}$ \\
9 & $\left(n_{1}, d_{1}\right)$ & 12.5 & 125.85 & 1.22 & $\mathrm{~B}^{+}$ \\
10 & $\left(n_{2}, d_{2}\right)$ & 13 & 124.58 & -0.64 & $\mathrm{~A}^{-}$ \\
11 & $\left(n_{1}, d_{1}\right)$ & 14.5 & 123.65 & -1.61 & $\mathrm{~B}^{-}$ \\
12 & $\left(n_{2}, d_{2}\right)$ & 15 & 123.67 & -2.51 & $\mathrm{C}^{-}$ \\
$\mathbf{1 3}$ & $\left(n_{2}, d_{2}\right)$ & $\mathbf{1 5 . 5}$ & $\mathbf{1 2 2 . 8 5}$ & $-\mathbf{4 . 1 7}$ & $\mathrm{D}^{-}$ \\
14 & $\left(n_{2}, d_{2}\right)$ & 16 & 123.20 & -3.46 & $\mathrm{C}^{-}$ \\
15 & $\left(n_{2}, d_{2}\right)$ & 16.5 & 123.90 & -2.03 & $\mathrm{C}^{-}$ \\
16 & $\left(n_{2}, d_{2}\right)$ & 17 & 122.98 & -3.90 & $\mathrm{D}^{-}$ \\
17 & $\left(n_{2}, d_{2}\right)$ & 17.5 & 123.52 & -2.81 & $\mathrm{C}^{-}$ \\
18 & $\left(n_{2}, d_{2}\right)$ & 18 & 123.72 & -2.41 & $\mathrm{C}^{-}$ \\
19 & $\left(n_{2}, d_{2}\right)$ & 18.5 & 124.00 & -1.83 & $\mathrm{C}^{-}$ \\
20 & $\left(n_{2}, d_{2}\right)$ & 19 & 123.87 & -2.10 & $\mathrm{C}^{-}$ \\
\hline
\end{tabular}

It is observed from either Table 9 or Figure 2 that the VSSI-Synth / VSSI-Runs monitoring scheme using a $1 \& 2$ strategy in steady-state mode issues the first OOC signal at sampling number 13 or cumulative time of 15.5 hours indicating an unexpected downwards shift in the mean weight of the yogurt cups, due to some undesired assignable cause(s). After some investigation, it was observed that it was due to a clog in the pipe of the machine.

\section{Conclusion}

Since autocorrelation and measurement errors are often encountered in real life applications of SPM; then in this paper, the proposed use of improved synthetic and runs-rules $\bar{X}$ schemes with an embedded VSSI approach is essential because they yield a significant improvement as compared to the existing FSSI $\bar{X}$ scheme. Moreover, to reduce the negative effect of autocorrelation and measurement errors, the $s \& m$ strategy does yield a desired effect of improving the OOC performance of the proposed monitoring schemes. Individually, the autocorrelation levels have a greater negative effect as compared to the measurement errors, and thus, the $s$-skip strategy has a greater improvement influence than the multiple measurement strategy. For small-to-moderate levels of autocorrelation, reasonable values of $s$ are sufficient; however, for large levels of autocorrelation and if the source dataset is limited, then the required values of $s$ may violate the concept of rational subgroups; but is fully applicable in a 'big data' environment. A drawback of these new monitoring schemes with $s \& m$ strategy is that, they require more observations (as some will be skipped during inspection) and more effort (multiple measurements on each item are taken during inspection) as compared to the no remedy approach. Finally, quality practitioners are recommended to implement the proposed improved synthetic and runs-rules $\bar{X}$ schemes with an embedded VSSI approach instead of the existing FSSI $\bar{X}$ scheme when monitoring the process mean under the combined effect of autocorrelation and measurement errors. For future research, the effect of autocorrelation and measurement errors on the memory-type monitoring schemes as well as on nonparametric monitoring schemes can be studied. Moreover, the performance of the schemes discussed herein when underlying process parameters are unknown need to be investigated. 


\section{References}

Adeoti, O.A., \& Malela-Majika, J.-C. (2020). Double exponentially weighted moving average control chart with supplementary runs-rules. Quality Technology \& Quantitative Management, 17(2), 149-172.

Alwan, L.C., \& Radson, D. (1992). Time-series investigation of subsample mean chart. IIE Transactions, 24(5), 66-80.

Aslam, M., \& Ali, M.M. (2019). Testing and Inspection using acceptance sampling plans. Springer Nature Pte Ltd., Singapore. DOI: 10.1007/978-981-13-9306-8.

Celano, G., Costa, A., \& Fichera, S. (2006). Statistical design of variable sample size and sampling interval $\bar{X}$ control charts with runs rules. International Journal of Advanced Manufacturing Technology, 28, 966-977.

Chew, X.Y., Khaw, K.W., \& Yeong, W.C. (2020). The efficiency of run rules schemes for the multivariate coefficient of variation: a Markov chain approach. Journal of Applied Statistics, 47(3), 460-480.

Costa, A.F.B. (1997). $\bar{X}$ chart with variable sample size and sampling interval. Journal of Quality Technology, 29(2), 197-204.

Costa, A.F.B., \& Castagliola, P. (2011). Effect of measurement error and autocorrelation on the $\bar{X}$ chart. Journal of Applied Statistics, 38(4), 661-673.

Costa, A.F.B., \& Machado, M.A.G. (2016). A side-sensitive synthetic chart combined with a VSS $\bar{X}$ chart. Computers \& Industrial Engineering, 91, 205-214.

Davis, R.B., \& Woodall, W.H. (2002). Evaluating and improving the synthetic control chart. Journal of Quality Technology, 34(2), 200-208.

Fu, J.C., \& Lou, W.Y.W. (2003). Distribution Theory of Runs and Patterns and Its Applications: A Finite Markov Chain Imbedding Approach. World Scientific Publishing, Singapore.

Haq, A. (2019). A new nonparametric synthetic EWMA control chart for monitoring process mean. Communications in Statistics - Simulation and Computation, 48(6), 1665-1676.

Haq, A., \& Khoo, M.B.C. (2019). A synthetic double sampling control chart for process mean using auxiliary information. Quality and Reliability Engineering International, 35(6), 1803-1825.

Hu, X.L., \& Sun, J. (2015). Synthetic $\bar{X}$ chart for AR(1) autocorrelated processes. Proceedings of the $27^{\text {th }}$ Chinese Control and Decision Conference, 7-12. DOI: 10.1109/CCDC.201527161658.

Jensen, W.A., Bryce, G.R., Reynolds Jr., M.R. (2008). Design issues for adaptive control charts. Quality and Reliability Engineering International, 24(4), 429-445.

Khaw, K.W., Chew, X.Y., Yeong, W.C., \& Lim, S.L. (2019). Optimal design of the synthetic control chart for monitoring the multivariate coefficient of variation. Chemometrics and Intelligent Laboratory Systems, $186,33-40$.

Khoo, M.B.C., See, M.Y., Chong, N.L., \& Teoh, W.L. (2019). An improved variable sample size and sampling interval $S$ control chart. Quality and Reliability Engineering International, 35(1), 392-404.

Koutras, M.V., Bersimis, S., \& Maravelakis, P.E. (2007). Statistical process control using Shewhart control charts with supplementary runs rules. Methodology and Computing in Applied Probability, 9(2), 207-224.

Lee, M.H., \& Khoo, M.B.C. (2018). Economic-statistical design of control chart with runs rules for correlation within sample. Communications in Statistics - Simulation and Computation, 47(10), 28492864.

Linna, K.W., \& Woodall, W.H. (2001). Effect of measurement error on Shewhart control charts. Journal of Quality Technology, 33(2), 213-222.

Mabude, K., Malela-Majika, J.-C., \& Shongwe S.C. (2020). A new distribution-free generally weighted moving average monitoring scheme for detecting unknown shifts in the process location. International Journal of Industrial Engineering Computations, 11(2), 235-254.

Maleki, M.R., Amiri, A., \& Castagliola, P. (2017). Measurement errors in statistical process monitoring: A literature review. Computers \& Industrial Engineering, 103, 316-329.

Malela-Majika, J.-C. (2019). Modified side-sensitive synthetic double sampling monitoring scheme for simultaneously monitoring the process mean and variability. Computers and Industrial Engineering, 130, 798-814.

Maravelakis, P.E., Castagliola, P., \& Khoo, M.B.C. (2019). Run length properties of run rules EWMA chart using integral equations. Quality Technology \& Quantitative Management, 16(2), 129-139. 
Mehmood, R., Lee, M.H., Hussain, S., \& Riaz, M. (2019). On efficient construction and evaluation of runs rules based $\bar{X}$ control chart for known and unknown parameters under different distributions. Quality and Reliability Engineering International, 35(2), 582-599.

Noorossana, R., Maryam, S.A., \& Deheshvar, A. (2015). Combined variable sample size, sampling interval and double sampling (CVSSIDS) adaptive control charts. Communications in Statistics - Theory and Methods, 44(6), 1255-1269.

Noorossana, R., Deheshvar, A., \& Maryam, S.A. (2016). A modified variable sample size and sampling interval control chart. International Journal of Advanced Manufacturing Technology, 84, 1303-1312.

Prabhu, S.S., Montgomery, D.C., \& Runger, G.C. (1994). A combined adaptive sample size and sampling interval $\bar{X}$ control scheme. Journal of Quality Technology, 26(3), 164-176.

Prajapati, D.R., \& Singh, S. (2012). Control charts for monitoring the autocorrelated process parameters: A literature review. International Journal of Productivity and Quality Management, 10(2), 207-249.

Psarakis, S. (2015). Adaptive control charts: Recent developments and extensions. Quality and Reliability Engineering International, 31(7), 1265-1280.

Rakitzis, A.C., Chakraborti, S., Shongwe, S.C., Graham, M.A., \& Khoo, M.B.C. (2019). An overview of synthetic-type control charts: Techniques and Methodology. Quality and Reliability Engineering International, 35(7), 2081-2096.

Raza, M.A., Nawaz, T., \& Han, D. (2019). On designing new optimal synthetic Tukey's control charts. Journal of Statistical Computation and Simulation, 89(12), 2218-2238.

Sabahno, H., \& Amiri, A. (2017). The effect of measurement errors on the performance of variable sample size and sampling interval $\bar{X}$ control chart. International Journal of Engineering, Transactions A: Basics, 30(7), 995-1004.

Shongwe S.C., \& Graham, M.A. (2016). On the performance of Shewhart-type synthetic and runs-rules charts combined with an $\bar{X}$ chart. Quality and Reliability Engineering International, 32(4), 1357-1379.

Shongwe, S.C., Malela-Majika, J.-C., Castagliola, P., \& Molahloe, T. (2019a). Side-sensitive synthetic and runs-rules charts for monitoring $\mathrm{AR}(1)$ processes with skipping sampling strategies. Communications in Statistics - Theory and Methods, DOI: 10.1080/03610926.2019.1596284.

Shongwe, S.C., Malela-Majika, J.-C., \& Rapoo, E.M. (2019b). One-sided and two-sided $w$-of-w runs-rules schemes: An overall performance perspective and the unified run-length derivations. Journal of Probability and Statistics, Article ID: 6187060, 1-20.

Shongwe, S.C., \& Malela-Majika, J.-C. (2019). Shewhart-type monitoring schemes with supplementary wof-w runs-rules to monitor the mean of autocorrelated samples. Communications in Statistics - Simulation and Computation, DOI: 10.1080/03610918.2019.1650180.

Shongwe, S.C., Malela-Majika, J.-C., and Castagliola, P. (2020a). The new synthetic and runs-rules schemes to monitor the process mean of autocorrelated observations with measurement errors. Communications in Statistics - Theory and Methods, DOI: 10.1080/03610926.2020.1737125.

Shongwe, S.C., Malela-Majika, J.-C., and Castagliola, P. (2020b). On monitoring the process mean of autocorrelated observations with measurement errors using the w-of-w runs-rules scheme. Quality and Reliability Engineering International, 36(3), 1144-1160.

Singh, S., \& Prajapati, D.R. (2014). Effect of warning limits on the performance of the $\bar{X}$ chart under autocorrelation. International Journal of Productivity and Quality Management, 13(2), 235-250.

Tran, P.H., Tran, K.P., \& Rakitzis, A.C. (2019). A synthetic median control chart for monitoring the process mean with measurement errors. Quality and Reliability Engineering International, 35(4), 1100-1116.

$\mathrm{Wu}, \mathrm{Z}$., \& Spedding, T.A. (2000). A synthetic control chart for detecting small shifts in the process mean. Journal of Quality Technology, 32(1), 32-38.

Yu, S., Wan, Q., Wei, Z., \& Tang, T. (2016). Statistical design of an adaptive synthetic $\bar{X}$ control chart with run rule on service and management operation. Scientific Programming, Article ID: 9629170, 1-7.

\section{Appendix: Transition probability matrix of the VSSI-Runs and VSSI-Synth schemes}

To construct a general transition probability matrices (TPM) for any monitoring scheme, the scheme's charting region need to be divided into separate distinct regions as shown in Figure 1 - this is consistent with the manner that it is done in Fu and Lou (2003)'s Chapter 4, Celano et al. (2006), Costa and Machado 
(2016), Shongwe and Graham (2016). That is, let $\left\{Z_{t} ; t \geq 0\right\}$ be a sequence of autocorrelated, with measurement errors, trials taking values within the set $\zeta=\left\{\mathrm{A}^{+}, \mathrm{A}^{-}, \mathrm{B}^{+}, \mathrm{B}^{-}, \mathrm{C}^{+}, \mathrm{C}^{-}, \mathrm{D}^{+}, \mathrm{D}^{-}\right\}$. Next, the probabilities of these events depend on the sample size (i.e. $n_{r}$ ) and sampling interval (i.e. $d_{r}$ ); that is, for $\left(n_{1} \& d_{1}\right)$, the probability that a plotting statistic falls in the regions $\mathrm{A}^{+}, \mathrm{A}^{-}, \mathrm{B}^{+}, \mathrm{B}^{-}, \mathrm{C}^{+}, \mathrm{C}^{-}, \mathrm{D}^{+}, \mathrm{D}^{-}$ is given by $\mathrm{A}_{1}^{+}, \mathrm{A}_{1}^{-}, \mathrm{B}_{1}^{+}, \mathrm{B}_{1}^{-}, \mathrm{C}_{1}^{+}, \mathrm{C}_{1}^{-}, \mathrm{D}_{1}^{+}, \mathrm{D}_{1}^{-}$, whereas for $\left(n_{2} \& d_{2}\right)$, the probabilities are given by $\mathrm{A}_{2}^{+}$, $\mathrm{A}_{2}^{-}, \mathrm{B}_{2}^{+}, \mathrm{B}_{2}^{-}, \mathrm{C}_{2}^{+}, \mathrm{C}_{2}^{-}, \mathrm{D}_{2}^{+}, \mathrm{D}_{2}^{-}$, respectively. For some $\left(n_{r} \& d_{r}\right)$, suppose that the values of $\mu_{0}$ and $\sigma_{0}$ are known, then these probabilities are given by

$$
\begin{aligned}
& \mathrm{A}_{r}^{+}=\Phi\left(k_{3}-\delta \sqrt{n_{r}}\right)-\Phi\left(-\delta \sqrt{n_{r}}\right), \\
& \mathrm{A}_{r}^{-}=\Phi\left(-\delta \sqrt{n_{r}}-\Phi\left(-k_{3}-\delta \sqrt{n_{r}}\right),\right. \\
& \mathrm{B}_{r}^{+}=\Phi\left(k_{2}-\delta \sqrt{n_{r}}\right)-\Phi\left(k_{3}-\delta \sqrt{n_{r}}\right), \\
& \mathrm{B}_{r}^{-}=\Phi\left(-k_{3}-\delta \sqrt{n_{r}}\right)-\Phi\left(-k_{2}-\delta \sqrt{n_{r}}\right), \\
& \mathrm{C}_{r}^{+}=\Phi\left(k_{1}-\delta \sqrt{n_{r}}\right)-\Phi\left(k_{2}-\delta \sqrt{n_{r}}\right), \\
& \mathrm{C}_{r}^{-}=\Phi\left(-k_{2}-\delta \sqrt{n_{r}}\right)-\Phi\left(-k_{1}-\delta \sqrt{n_{r}}\right), \\
& \mathrm{D}_{r}^{+}=1-\Phi\left(k_{1}-\delta \sqrt{n_{r}}\right), \mathrm{D}_{r}^{-}=\Phi\left(-k_{1}-\delta \sqrt{n_{r}}\right), \\
& \mathrm{A}_{r}=\mathrm{A}_{r}^{+}+\mathrm{A}_{r}^{-}, \mathrm{B}_{r}=\mathrm{B}_{r}^{+}+\mathrm{B}_{r}^{-}, \mathrm{C}_{r}=\mathrm{C}_{r}^{+}+\mathrm{C}_{r}^{-}, \mathrm{D}_{r}=\mathrm{D}_{r}^{+}+\mathrm{D}_{r}^{-} .
\end{aligned}
$$

The TPM of the Markov chain technique for any positive integer value of $M>0$ is given by a $(M+$ 1) $\times(M+1)$ matrix $\mathbf{P}$,

$$
\mathbf{P}=\left(\begin{array}{cc}
\mathbf{Q} & \mathbf{r} \\
\mathbf{0}^{\prime} & 1
\end{array}\right)
$$

where $\mathbf{Q}$ is the $M \times M$ essential TPM with its elements given by the probabilities in Equation (A1), the $M \times 1$ vector $\mathbf{r}$ satisfies $\mathbf{r}=\mathbf{1}-\mathbf{Q 1}$ with the $M \times 1$ vectors given by $\mathbf{1}=\left(\begin{array}{llll}1 & 1 & \ldots & 1\end{array}\right)^{\mathrm{T}}$ and $\mathbf{0}=$ $\left(\begin{array}{llll}0 & 0 & \ldots & 0\end{array}\right)^{\mathrm{T}}$. To define the run-length characteristics, the compound patterns that result in an OOC event (which is also known as the waiting time until the first occurrence of an OOC signal) need to be defined. For example, the sequence of plotting statistics ${ }^{\prime} \mathrm{C}^{+} \mathrm{C}^{+}$' indicates two consecutive plotting statistics falling in region $\mathrm{C}^{+}$. The symbol ' \pm ' is used to denote the assumption that (at time 0 ) the first observation falls either in region $\mathrm{C}^{+}$or in region $\mathrm{C}^{-}$, i.e. the head-start feature for the synthetic monitoring schemes (see Davis and Woodall (2002)); so that ' $\pm \mathrm{C}^{-}$' indicate that the first plotting statistic, at time 0, falls either in region $\mathrm{C}^{+}$or $\mathrm{C}^{-}$and the second in region $\mathrm{C}^{-}$. Define $\Lambda$ as a compound pattern if it is the union of $\omega$ distinct simple absorbing patterns i.e. $\Lambda=\Lambda_{1} \cup \Lambda_{2} \cup \ldots \cup \Lambda_{\omega}$. Similarly, define $\Psi$ as a compound pattern if it is the union of $v$ distinct simple absorbing patterns with a head-start states i.e. $\Psi=\Psi_{1} \cup$ $\Psi_{2} \cup \ldots \cup \Psi_{v}$. Let $W$ denote the waiting time for the first occurrence of either $\Lambda$ or $\Psi-$ as these are the absorbing states of the Markov chain. Then the run-length distribution of the VSSI-Runs and VSSI-Synth $\bar{X}$ monitoring schemes coincides with the waiting time distribution of $W$. Hence, the Markov chain matrix with the state space, $\Omega$, operating on $\left\{Z_{t}\left(n_{r}, d_{r}\right) ; t \geq 0\right\}$ is as follows:

- Absorbing states (denoted by 'OOC') - the union of $\Lambda_{1}, \ldots, \Lambda_{\omega}, \Psi_{1}, \ldots, \Psi_{v}$, i.e. the $\omega+v$ states that signal the entrance of the Markov chain to an absorbing state;

- Sub-patterns (denoted by $\eta_{1}, \ldots, \eta_{\tau}$ and $\psi_{1}, \ldots, \psi_{\kappa}$ ) - the distinct first element(s) of the simple pattern $\Lambda_{1}, . ., \Lambda_{\omega}$ and $\Psi_{1}, . ., \Psi_{v}$ without the last element, where $\tau<\omega$ and $\kappa<v$, respectively. For instance, if $\Lambda_{1}=\left\{\mathrm{C}^{+} \mathrm{B}^{+} \mathrm{C}^{+}\right\}$then $\eta_{1}=\left\{\mathrm{C}^{+} \mathrm{B}^{+}\right\}$, similarly, if $\Psi_{1}=\left\{ \pm \mathrm{B}^{+} \mathrm{C}^{+}\right\}$then $\psi_{1}=\left\{ \pm \mathrm{B}^{+}\right\}$;

- Central regions - two of the $\eta_{i}$ 's are equal to the transient states, denoted by $\varphi_{a}$ and $\varphi_{b}$, corresponding to the IC central regions for $\left(n_{1} \& d_{1}\right)$ and $\left(n_{2} \& d_{2}\right)$, respectively.

For illustration purpose, the construction of the TPMs of the VSSI-Synth and VSSI-Runs monitoring schemes when $H=1$ and 2 are shown in detail. In Table A1, the compound patterns (i.e. $\Lambda$ and $\Psi$ ), their corresponding sub-patterns (i.e. $\eta$ and $\psi$ ) and the corresponding state spaces are shown when $H=1$ and 2. Note that the VSSI-Runs schemes exclude the compound (and sub-patterns) that corresponds to $\Psi$ (and $\psi$ ), respectively. Next, Equations (A1) and (A2) are used to construct the corresponding TPMs shown in Table A2, when $H=1$ and 2. 


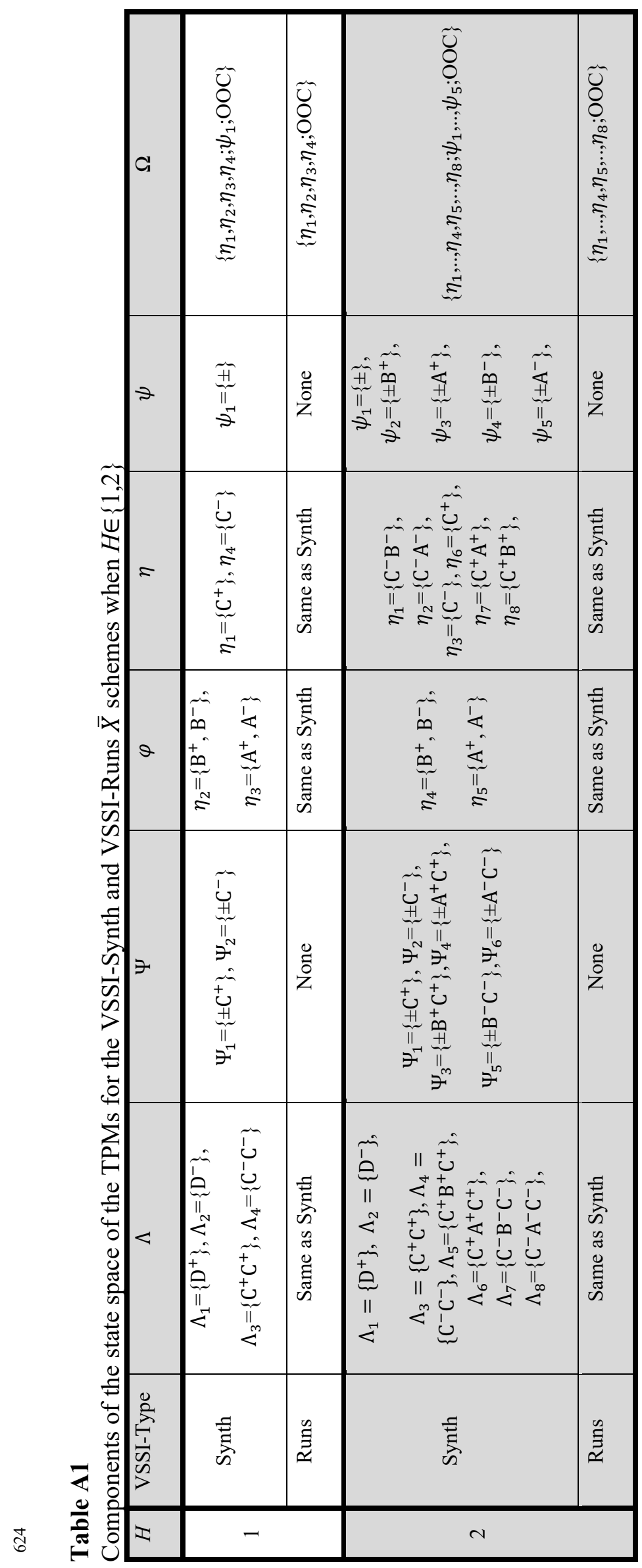


Table A2

TPMs of the VSSI-Synth and VSSI-Runs schemes when $H \in\{1,2\}$

H=1: VSSI-Synth (removing $\psi_{1}$ yields VSSI-Runs) scheme

\begin{tabular}{c|ccccc|c} 
& $\eta_{2}$ & $\eta_{3}$ & $\eta_{4}$ & $\psi_{1}$ & \\
\hline$\eta_{1}$ & $\eta_{1}$ & $\mathrm{~B}_{2}^{-}+\mathrm{B}_{2}^{+}$ & $\mathrm{A}_{2}^{-}+\mathrm{A}_{2}^{+}$ & $\mathrm{C}_{2}^{+}$ & $\mathrm{COC}_{2}^{-}+\mathrm{D}_{2}^{-}+\mathrm{D}_{2}^{+}$ \\
$\eta_{2}$ & $\mathrm{C}_{2}^{-}$ & $\mathrm{B}_{2}^{-}+\mathrm{B}_{2}^{+}$ & $\mathrm{A}_{2}^{-}+\mathrm{A}_{2}^{+}$ & $\mathrm{C}_{2}^{+}$ & $\mathrm{D}_{2}^{-}+\mathrm{D}_{2}^{+}$ \\
$\eta_{3}$ & $\mathrm{C}_{1}^{-}$ & $\mathrm{B}_{1}^{-}+\mathrm{B}_{1}^{+}$ & $\mathrm{A}_{1}^{-}+\mathrm{A}_{1}^{+}$ & $\mathrm{C}_{1}^{+}$ & $\mathrm{D}_{1}^{-}+\mathrm{D}_{1}^{+}$ \\
$\eta_{4}$ & $\mathrm{C}_{2}^{-}$ & $\mathrm{B}_{2}^{-}+\mathrm{B}_{2}^{+}$ & $\mathrm{A}_{2}^{-}+\mathrm{A}_{2}^{+}$ & & $\mathrm{C}_{2}^{+}+\mathrm{D}_{2}^{-}+\mathrm{D}_{2}^{+}$ \\
$\psi_{1}$ & & $\mathrm{~B}_{2}^{-}+\mathrm{B}_{2}^{+}$ & $\mathrm{A}_{2}^{-}+\mathrm{A}_{2}^{+}$ & & $\mathrm{C}_{2}^{-}+\mathrm{C}_{2}^{+}+\mathrm{D}_{2}^{-}+\mathrm{D}_{2}^{+}$ \\
& & & & 1 \\
\hline
\end{tabular}

$\mathbf{H = 2}$ : VSSI-Synth (removing $\psi_{1}, . ., \psi_{5}$ yields VSSI-Runs)

\begin{tabular}{|c|c|c|c|c|c|c|c|c|c|c|c|c|c|c|}
\hline & $\eta_{1}$ & $\eta_{2}$ & $\eta_{3}$ & $\eta_{4}$ & $\eta_{5}$ & $\eta_{6}$ & $\eta_{7}$ & $\eta_{8}$ & $\psi_{1}$ & $\psi_{2}$ & $\psi_{3}$ & $\psi_{4}$ & $\psi_{5}$ & OOC \\
\hline$\eta_{1}$ & & & & $\mathrm{~B}_{2}^{-}+\mathrm{B}_{2}^{+}$ & $A_{2}^{-}+A_{2}^{+}$ & $\mathrm{C}_{2}^{+}$ & \multirow{8}{*}{$\mathrm{A}_{2}^{+}$} & \multirow{8}{*}{$\mathrm{B}_{2}^{+}$} & & \multirow{14}{*}{$\mathrm{B}_{2}^{+}$} & \multirow{14}{*}{$\mathrm{A}_{2}^{+}$} & \multirow{14}{*}{$\mathrm{B}_{2}^{-}$} & \multirow{14}{*}{$\mathrm{A}_{2}^{-}$} & $\mathrm{C}_{2}^{-}+\mathrm{D}_{2}^{-}+\mathrm{D}_{2}^{+}$ \\
\hline$\eta_{2}$ & & & & $\mathrm{~B}_{1}^{-}+\mathrm{B}_{1}^{+}$ & $\mathrm{A}_{1}^{-}+\mathrm{A}_{1}^{+}$ & $\mathrm{C}_{1}^{+}$ & & & & & & & & $\mathrm{C}_{1}^{-}+\mathrm{D}_{1}^{-}+\mathrm{D}_{1}^{+}$ \\
\hline$\eta_{3}$ & $\mathrm{~B}_{2}^{-}$ & $A_{2}^{-}$ & & $\mathrm{B}_{2}^{+}$ & $\mathrm{A}_{2}^{+}$ & $\mathrm{C}_{2}^{+}$ & & & & & & & & $\mathrm{C}_{2}^{-}+\mathrm{D}_{2}^{-}+\mathrm{D}_{2}^{+}$ \\
\hline$\eta_{4}$ & & & $\mathrm{C}_{2}^{-}$ & $\mathrm{B}_{2}^{-}+\mathrm{B}_{2}^{+}$ & $\mathrm{A}_{2}^{-}+\mathrm{A}_{2}^{+}$ & $\mathrm{C}_{2}^{+}$ & & & & & & & & $\mathrm{D}_{2}^{-}+\mathrm{D}_{2}^{+}$ \\
\hline$\eta_{5}$ & & & $\mathrm{C}_{1}^{-}$ & $\mathrm{B}_{1}^{-}+\mathrm{B}_{1}^{+}$ & $\mathrm{A}_{1}^{-}+\mathrm{A}_{1}^{+}$ & $\mathrm{C}_{1}^{+}$ & & & & & & & & $\mathrm{D}_{1}^{-}+\mathrm{D}_{1}^{+}$ \\
\hline$\eta_{6}$ & & & $\mathrm{C}_{2}^{-}$ & $\mathrm{B}_{2}^{-}$ & $\mathrm{A}_{2}^{-}$ & & & & & & & & & $\mathrm{C}_{2}^{+}+\mathrm{D}_{2}^{-}+\mathrm{D}_{2}^{+}$ \\
\hline$\eta_{7}$ & & & $\mathrm{C}_{1}^{-}$ & $\mathrm{B}_{1}^{-}+\mathrm{B}_{1}^{+}$ & $\mathrm{A}_{1}^{-}+\mathrm{A}_{1}^{+}$ & & & & & & & & & $\mathrm{C}_{1}^{+}+\mathrm{D}_{1}^{-}+\mathrm{D}_{1}^{+}$ \\
\hline$\eta_{8}$ & & & $\mathrm{C}_{2}^{-}$ & $\mathrm{B}_{2}^{-}+\mathrm{B}_{2}^{+}$ & $\mathrm{A}_{2}^{-}+\mathrm{A}_{2}^{+}$ & & & & & & & & & $\mathrm{C}_{2}^{+}+\mathrm{D}_{2}^{-}+\mathrm{D}_{2}^{+}$ \\
\hline$\psi_{1}$ & & & \multirow{6}{*}{$\begin{array}{l}\mathrm{C}_{2}^{-} \\
\mathrm{C}_{1}^{-}\end{array}$} & & & & & & & & & & & $\mathrm{C}_{2}^{-}+\mathrm{C}_{2}^{+}+\mathrm{D}_{2}^{-}+\mathrm{D}_{2}^{+}$ \\
\hline$\psi_{2}$ & & & & $\mathrm{~B}_{2}^{-}+\mathrm{B}_{2}^{+}$ & $A_{2}^{-}+A_{2}^{+}$ & & & & & & & & & $\mathrm{C}_{2}^{+}+\mathrm{D}_{2}^{-}+\mathrm{D}_{2}^{+}$ \\
\hline$\psi_{3}$ & & & & $\mathrm{~B}_{1}^{-}+\mathrm{B}_{1}^{+}$ & $\mathrm{A}_{1}^{-}+\mathrm{A}_{1}^{+}$ & & & & & & & & & $\mathrm{C}_{1}^{+}+\mathrm{D}_{1}^{-}+\mathrm{D}_{1}^{+}$ \\
\hline$\psi_{4}$ & & & & $\mathrm{~B}_{2}^{-}+\mathrm{B}_{2}^{+}$ & $\mathrm{A}_{2}^{-}+\mathrm{A}_{2}^{+}$ & $\mathrm{C}_{2}^{+}$ & & & & & & & & $\mathrm{C}_{2}^{-}+\mathrm{D}_{2}^{-}+\mathrm{D}_{2}^{+}$ \\
\hline$\psi_{5}$ & & & & $\mathrm{~B}_{1}^{-}+\mathrm{B}_{1}^{+}$ & $\mathrm{A}_{1}^{-}+\mathrm{A}_{1}^{+}$ & $\mathrm{C}_{1}^{+}$ & & & & & & & & $\mathrm{C}_{1}^{-}+\mathrm{D}_{1}^{-}+\mathrm{D}_{1}^{+}$ \\
\hline $\mathrm{OOC}$ & & & & & & & & & & & & & & 1 \\
\hline
\end{tabular}

Continuing in the similar fashion, for other values of $H$, the state space of each of the VSSI-Synth and VSSI-Runs monitoring schemes is as follows,

VSSI-Synth: $\Omega=\left\{\eta_{1}, \ldots, \eta_{\frac{\tau}{2}-1} ; \eta_{\frac{\tau}{2}}=\varphi_{a}, \eta_{\frac{\tau}{2}+1}=\varphi_{b} ; \eta_{\frac{\tau}{2}+2}, \ldots, \eta_{\tau} ; \psi_{1}, \ldots, \psi_{\kappa} ; \mathrm{OOC}\right\}$,
VSSI-Runs: $\Omega=\left\{\eta_{1}, \ldots, \eta_{\frac{\tau}{2}-1} ; \eta_{\frac{\tau}{2}}=\varphi_{a}, \eta_{\frac{\tau}{2}+1}=\varphi_{b} ; \eta_{\frac{\tau}{2}+2}, \ldots, \eta_{\tau} ;\right.$ OOC $\}$,

where

$\tau=4 H$ and $\kappa=4 H-3$.

That is, removing the $\psi_{1}, \ldots, \psi_{\kappa}$ elements (corresponding to the head-start feature) on the state space of the VSSI-Synth scheme yields the state space of the VSSI-Runs scheme. Therefore, the dimension of the essential TPMs is given by $M=\tau+\kappa$. Note that in the steady-state mode, VSSI-Synth scheme's headstart feature is no longer applicable, and thus, the head-start elements on the TPM are discarded and consequently, the VSSI-Synth scheme's TPM is exactly the same as the VSSI-Runs scheme's TPM. In turn, the latter imply that the design parameters are also the same. This was first shown in Davis and Woodall (2002) for the FSSI schemes and re-iterated more recently in a review paper by Rakitzis et al. (2019). For example, the general TPM of the VSSI-Runs scheme (in both zero- and steady-state modes) and the VSSI-Synth scheme (in steady-state mode only) is given in Table A3. 


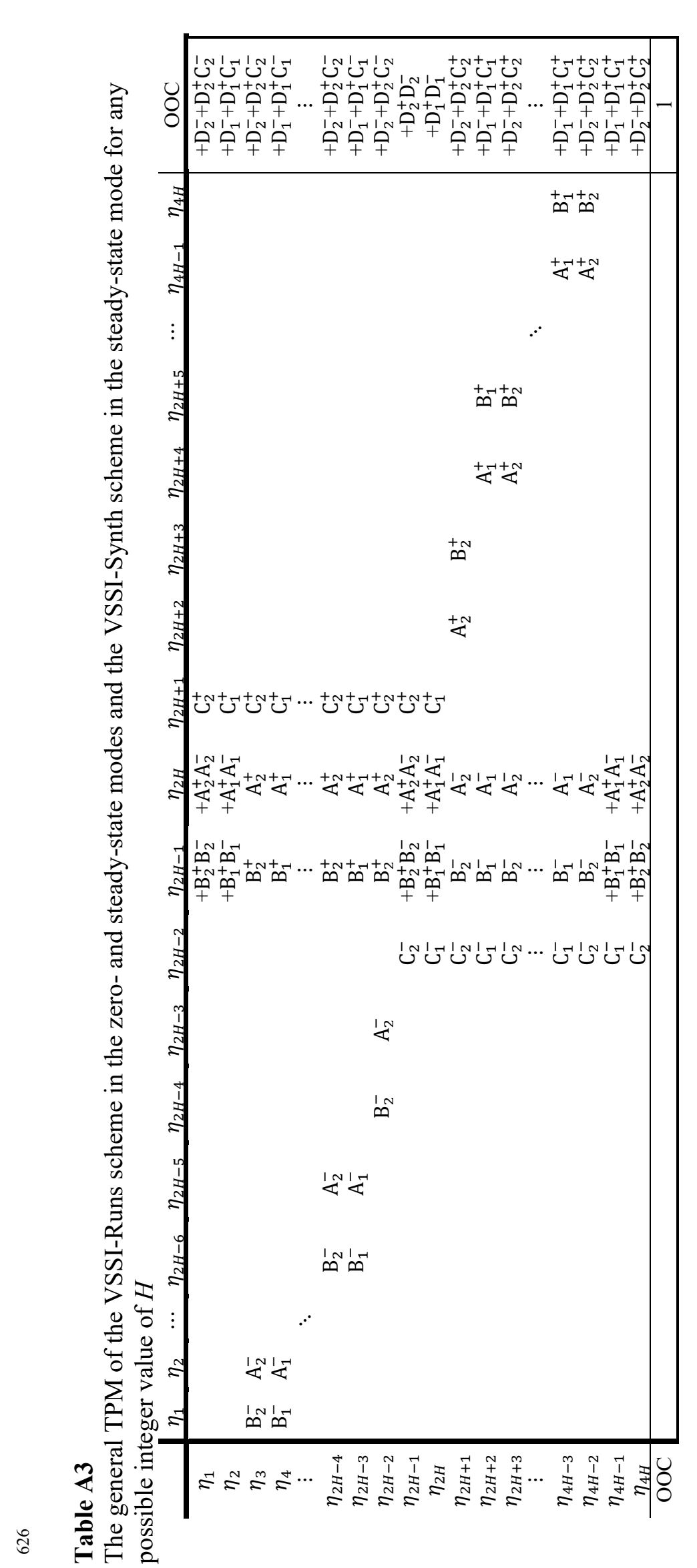

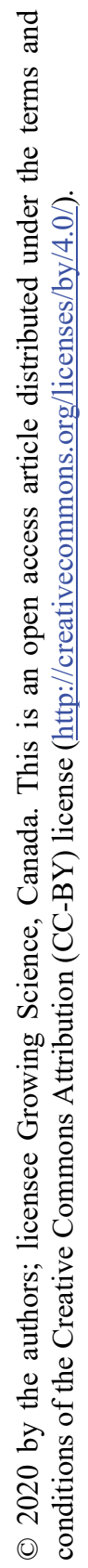

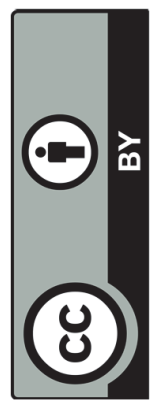

